\title{
WestVirginiaUniversity
}

THE RESEARCH REPOSITORY @ WVU

Graduate Theses, Dissertations, and Problem Reports

2016

\section{Analysis of curved composite beam}

\author{
Manish Aggarwal
}

Follow this and additional works at: https://researchrepository.wvu.edu/etd

\section{Recommended Citation}

Aggarwal, Manish, "Analysis of curved composite beam" (2016). Graduate Theses, Dissertations, and Problem Reports. 5033.

https://researchrepository.wvu.edu/etd/5033

This Thesis is protected by copyright and/or related rights. It has been brought to you by the The Research Repository @ WVU with permission from the rights-holder(s). You are free to use this Thesis in any way that is permitted by the copyright and related rights legislation that applies to your use. For other uses you must obtain permission from the rights-holder(s) directly, unless additional rights are indicated by a Creative Commons license in the record and/ or on the work itself. This Thesis has been accepted for inclusion in WVU Graduate Theses, Dissertations, and Problem Reports collection by an authorized administrator of The Research Repository @ WVU. For more information, please contact researchrepository@mail.wvu.edu. 


\title{
ANALYSIS OF CURVED COMPOSITE BEAM
}

\section{MANISH AGGARWAL}

\author{
Thesis submitted \\ to the Benjamin M. Statler College of Engineering and Mineral Resources \\ at the West Virginia University
}

In partial fulfilment of the requirements for the degree of

Master of Science in

Mechanical Engineering

Nithi T. Sivaneri, Committee Chair, Ph.D.

Victor H. Mucino, Ph.D.

Osama M. Mukdadi, Ph.D.

Department of Mechanical and Aerospace Engineering

Morgantown, West Virginia

December 2016

Keywords: Curved Composite Beam, h-p version finite element, Classical Laminate Plate Theory

Copyright 2016 Manish Aggarwal 


\section{ABSTRACT \\ ANALYSIS OF CURVED COMPOSITE BEAMS \\ MANISH AGGARWAL}

Composite materials are steadily replacing traditional materials in many engineering applications due to several benefits such as high strength to weight ratio and the ability to tailor the material for specific purposes. Over the last several decades the analysis of straight beams has received considerable attention while there is very little focus on curved composite beams.

In the present study, the formulation of the bending of a curved composite beam is based on the bending theory of thick shells. A variational formulation is employed to derive the governing equations. A consistent methodology is applied to reduce the two-dimensional nature of the composite constitutive equations (based on the classical laminate plate theory) to one dimension to reflect the nature of behaviour of a curved beam. In order to generate very accurate distributions of the stresses and strains in the curved beam, a higher-order finite element method ( $h-p$ version) is formulated. A unique curved-beam finite element is proposed.

A MATLAB code is written to carry out the numerical implementation of the composite curved beam problem. Results in the form of tangential stress distributions across the cross section and force and displacement distributions along the curved length of the beam are presented. The geometry of the composite curved beams considered consists of circular arcs. The study encompasses different types of loads and symmetric and unsymmetric layups. 


\section{ACKNOWLEDGEMENT}

I would like to thank my advisor, Dr. Nithi Sivaneri, from the bottom of my heart for providing guidance throughout my graduate education at WVU. I deeply value his time, inputs in developing a methodology which has never been tried before. I have learnt a lot from him, both professionally and personally. His suggestions have helped me a lot in improving my problem solving skills and explore new ways for implementing solutions.

I would also like to thank Dr. Victor Mucino for helping me during my entire graduate education life and Dr. Mukdadi for his time and valuable inputs for my thesis. I am very glad to be around wonderful people during my stay at WVU.

I am indebted to my parents, Asha and Arun, my brother Manu and my aunt and uncle, Savita and Krishan Jindal for their unconditional love and support. Although the professional challenges were inspiring and motivating it was only because of their support that I could overcome the personal challenges. Last but not the least are my friends Suvineeth, Nupur, Sriparna, Chetan, Akshay and Christina for being with me through the thick and thin of my graduate student life whom I can never forget because they made me smile. 
TABLE OF CONTENT

$\begin{array}{lr}\text { ABSTRACT } & \text { ACKNOWLEDGEMENT_ } \\ \text { TABLE OF CONTENT } & \text { iii } \\ \text { LIST OF FIGURES } & \text { iv } \\ \text { LIST OF TABLES } & \text { vi } \\ \text { INTRODUCTION_ } & \text { vii } \\ \end{array}$

1.1 Introduction 1

1.2 Laminated Composites___ 1

1.3 Literature Review _ 2

1.4 Need for Present Research _ 5

1.5 Objectives__ 5

1.6 Thesis Overview _ 6

2. THEORITICAL FORMULATION

2.1 Introduction 7

2.2 Coordinate System _ 7

2.3 Composite Lay-up Configuration __ 8

2.4 Plate Bending Theory __ 8

2.5 Kinematic Equations__ 9

2.6 Virtual Strain Energy __ 13

2.7 Composite Beam Formulation using Classical Laminate Plate Theory___ 17

2.7.1 Composite Beam Formulation for Thin Curved Beam __ 18

2.7.2 Composite Beam Formulation for Higher Order Term — 22

3. FINITE ELEMENT FORMULATION__ 29

3.1 Introduction 29

3.2 Finite Element Methods__ 29

3.3 Finite Element Shape Functions__ 30 
3.4 Element Stiffness Formulation using CLPT___ 34

3.4.1 Stiffness Matrix for a Thin Curved Beam Element ___ 36

3.4.2 Stiffness Matrix for Thick Curved Beam __ 39

4. NUMERICAL IMPLEMENTATION __ 42

4.1 Introduction __ 42

4.2 Numerical Integration in Space Domain __ 42

4.3 Boundary conditions __ 43

4.4 Ply Stresses for Beams ___ 43

5. RESULTS AND DISCUSSION ___ 46

5.1 Introduction _ 46

5.2 Verification of Curved Beam Results ___ 46

5.2.1 Isotropic Beam Subjected to Point Load __ 46

5.2.1.1 Radial Displacement ___ 48

5.2.1.2 Tangential Displacement___ 49

5.2.1.3 Reaction Forces___ 50

5.2.2 Isotropic Beam Subjected to Bending Moment ___ 51

5.2.3 Composite Pinched Ring____ 54

5.2.4 Stresses in a Curved Composite Beam Subjected to Bending Moment___ 56

5.3 Present Results__ 58

6. CONCLUSIONS \& RECOMMENDATIONS ___ 61

6.1 Contributions __ 61

6.2 Conclusions___ 61

6.3 Recommendations ___ 61

REFERENCES _ 63 


\section{LIST OF FIGURES}

Fig. 2-1 Curved beam segment ............................................................................................ 7

Fig 2-2 Composite Layup Configuration (Barbero, 2010) ....................................................... 8

Fig 3-1: Element definition for reduced beam formulation using CLPT ................................. 30

Fig 5-1: Curved beam subjected to bending moment and its cross section. (Nguyen, 2010)...... 52

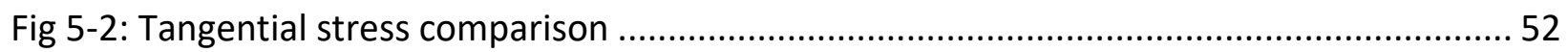

Fig 5-3: Composite pinched ring under compressive point load. (Kim, 2005) .......................... 55

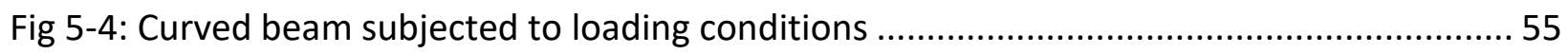

Fig 5.5: Tangential Stress plot along the varying Radial Position .......................................... 58 


\section{LIST OF TABLES}

Table 4:1: Sampling points and weights for seven-point Gauss Quadrature

Table 5:1 Radial displacement for first order formulation ............................................. 48

Table 5:2 Radial displacement for Second order formulation............................................. 49

Table 5:3 Tangential displacement for first order formulation ........................................ 49

Table 5:4 Tangential displacement for second order formulation ...................................... 50

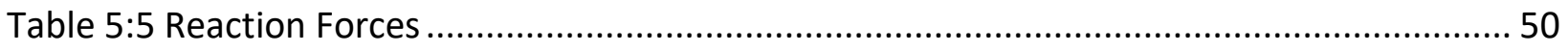

Table 5:6: Tangential Stress Values for Isotropic Material ............................................. 53

Table 5:7 Comparison of normalized shear force in pinched ring ....................................... 56

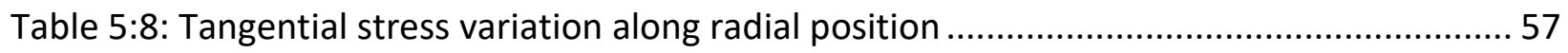

Table 5:9: Maximum deflection and moment of simply supported curved beam First Order .... 59

Table 5:10: Maximum deflection and moment of simply supported curved beam Second Order 59

Table 5:11: Maximum deflection and moment of simply supported curved beam First Order .. 60 Table 5:12: Maximum deflection and moment of simply supported curved beam Second Order 


\section{INTRODUCTION}

\subsection{Introduction}

Beams are one of the most fundamental structural components. Composite beams are lightweight and can be found in many diverse applications such as aerospace, medical, automobile and construction industries. Steel framed structures, buildings are the most common civil engineering applications. In such applications, beams are used as structural elements or components that support the whole structure. Also, the whole structure at a preliminary level can be modelled as a beam. In mechanical engineering, several parts such as rotating shafts carrying pulleys, gears are examples of beams. In the automobile industry, frames in vehicles (e.g., a truck) are beams are a unique example for beams. Robotic arms in manufacturing are modeled as beams as well. In aerospace engineering, beams (curved and straight) are found in many areas of an airplane or a space vehicle. In addition, the whole wing of a plane is often modeled as a beam for some preliminary analysis (Hajianmaleki \& Qatu, 2011).

Curved beams have been extensively used in various engineering applications. Naturallycurved or twisted beams have been a classical problem in theory of elasticity and several papers have been written to this subject to address their behaviour under varying conditions (Washizu, 1964).

\subsection{Laminated Composites}

The main phases of a composite material are fibres and matrix. While the fibre provides most of the stiffness and strength to a composite structure, the matrix binds the fibres together enabling them to transfer loads among fibres; further the matrix is responsible for shear strength 
and in-plane transverse strength. Composite materials have superior properties such as light weight, high strength to weight ratio, good corrosion and fatigue resistance and ease of manufacturing, compared to the traditional isotropic materials like steel, aluminium and orthotropic materials like concrete and wood.

Several theories have been applied to analyse complicated composite structures. Due to their large planar dimensions in comparison to the thickness, the composite structures can be treated as plate elements and therefore plate theories are adopted in analyzing such composite structures. The plate theories can be reduced to beam theories to analyse beams made of composite materials.

\subsection{Literature Review}

Numerous researches have studies the linear and nonlinear response of straight beams but there has not been substantial work related to curved beams.

Laminated composites are usually treated as plate elements because they have their planar dimensions larger than the thickness dimension. Several laminate theories have been developed for the analysis of plates made of composite laminates; the simplest one is the Classical Laminate Plate Theory (CLPT). The major drawback for CLPT is that the transverse shear strains are ignored and of the transverse shear effects may play an important role even for thin composite plates since the shear modulus of composites is much lower than their isotropic counterparts. Two prominent approaches to account for the transverse shear deformation are the First Order Shear Deformation Theory (FSDT) and Higher Order Shear Deformation Theory. An excellent description of these composite plate theories can be found in Reddy (2007). 
(Borri, et al., 1992) present a theory for the deformation of a naturally-curved and twisted anisotropic beam. The proposed theory extends the classical Saint-Venant Principle to curved and twisted anisotropic beams. Their model is for a curved beam of uniform cross section and assumes that the curvature and twist form elastic coupling due to the material properties and the curved geometry.

(Tanov \& Tabiei, 2000) have represented the transverse shear effects in a Mindlin-type displacement-based shell finite elements. Their formulation uses robust first order shear deformation shell finite element that eliminates the need for a shear correction factor.

(Kim, 2005) has presented a highly-accurate composite laminated hybrid-mixed curved beam element. The element formulation is based on the Hellinger-Reissner variational principle and employs the first-order shear deformation theory. Kim resorts to some nodeless degrees of freedom considers as an example a composite laminated pinched ring under compressive point load.

(Ecsedi \& Dluhi, 2005) have presented a one-dimensional mechanical model to analyse the static and the dynamic response of non-homogeneous symmetrical-cross-section curved beams and closed rings. They determine two kinematic variables to express the equation of motion and the boundary conditions, one being the radial displacement of the cross sections and the other being the rotation of the cross-section. They use the classical Bernoulli-Euler beam theory to find out the displacements which are confirmed to be exact for the various examples that they have illustrated in the paper.

(Erkmen \& Bradford, 2009) have developed a 3-D elastic total Lagrangian formulation for analysing a steel-concrete curved beam. Geometric nonlinearities are used to derive the strain 
expressions, the partial interaction at the interface in the tangential and radial directions due to flexible shear connectors. They present examples that illustrate the effects of curvature and geometric nonlinearity. They compare their results with a model based on the ABAQUS shell elements. Their formulation is very accurate and effective in determining the behaviour of the curved in-plan composite beam.

(Nguyen, 2010) has studied the variation of tangential stresses and radial stresses using a 3D finite element model of curved laminated beam. He studied the behaviour of the beam using isotropic and orthotropic material properties. Although the paper proves its results analytically but the tangential stress distribution along the thickness for an orthotropic material is a linear, distribution which is only possible if a continuum orthotropic configuration is considered.

(Hajianmaleki \& Qatu, 2012) have employed the FSDT with modified $\boldsymbol{A B D}$ matrices to analyze the static and free vibration behaviour of a curved beam. Their results are in the form of deflections, moment resultants, and natural frequencies. They obtain exact solutions and numerical solutions using general differential quadrature for several different boundary conditions. The results obtained are compared with that of an ANSYS model and they clearly show that the FSDT can accurately predict the static and free vibration behaviour for different lay-ups and boundary conditions.

(Tornabene, et al., 2013) have investigated the static behaviour of doubly-curved laminated composite shells and panels and proposed a general higher-order equivalent single-layer approach based on a Carrera unified formulation. They have derived a generalized differential quadrature numerical methodology to analyse the structures. To calculate quantities such as displacements, strains, and stresses, a three-dimensional stress-recovery procedure based on 
shell equilibrium equations is used. They have presented several loading conditions, lamination schemes, and boundary conditions and the results from these have been compared to the results obtained using a commercial finite element code.

\subsection{Need for Present Research}

The advancement in the field of composite material has replaced conventional materials in many appropriate applications. Numerous studies have been made in regards to straight composite beams under static and dynamic conditions; on the other hand, research pertaining to curved composite beams is limited. Most researchers of curved beam formulation just simply set all the strain and curvature components associated with the $y$ direction to be zero. This practice may be passable for isotropic curved beams even though the completely neglecting the Poisson's effect is questionable; for composite beams the transverse strains and the associated elastic constants play an important role even in the case of a beam when one dimension is larger than the cross section dimensions. In the present research when reducing the shell equations to that of a curved beam, we do not set the strains and curvatures associated with the $y$ direction to be zero; instead they are eliminated using the process of static condensation.

have assumed the

\subsection{Objectives}

The objectives of the thesis are:

- To formulate the problem of a composite curved beam applying the bending theory of shells and CLPT. 
- To apply the principle of virtual work for the above formulation.

- To formulate an $h-p$ version finite element model for a curved composite beam.

- To generate a MATLAB code to solve the finite element equations of the composite curved beam.

- To obtain results in the form of displacements, forces and stresses at any cross section along the length of the beam.

\subsection{Thesis Overview}

Chapter two deals with the coordinate system, composite lay-up configuration, loading configurations, introduction to plate theories, formulation of the governing equations and the systematic reduction of shell theory to a one-dimensional curved beam.

Chapter three details the finite element formulation of the shape functions, the stiffness matrix, and the load vector for the $h-p$ version finite element model.

Chapter four presents the numerical integration scheme in space domain using Gaussian quadrature, boundary conditions, the procedure for determining the ply stresses for curved beams using CLPT.

Chapter five contains the results of several examples of composite curved beams.

Chapter six contains the conclusions of the present work and recommendations for future work. 


\section{THEORITICAL FORMULATION}

\subsection{Introduction}

This chapter explains the theoretical formulation for the analysis of curved composite beams which is subjected to static loading conditions. The equations that govern this formulation are discussed in this chapter along with basic laminate theory which is the Classical Laminate Plate Theory (CLPT).

\subsection{Coordinate System}

Consider a cylindrical curved beam segment of angle $\alpha$, mid-surface radius $R$, thickness $h$, and width $b$ made of a composite laminate as shown in Fig. 2.1. The origin of the Cartesian coordinate system $x y z$ is at the left end of the segment at the mid surface with the $x$ axis tangential to the mid-surface line, the $y$ axis along the width, and the $z$ axis along the radial line. The angular coordinate $\theta$ is measured from the left end.

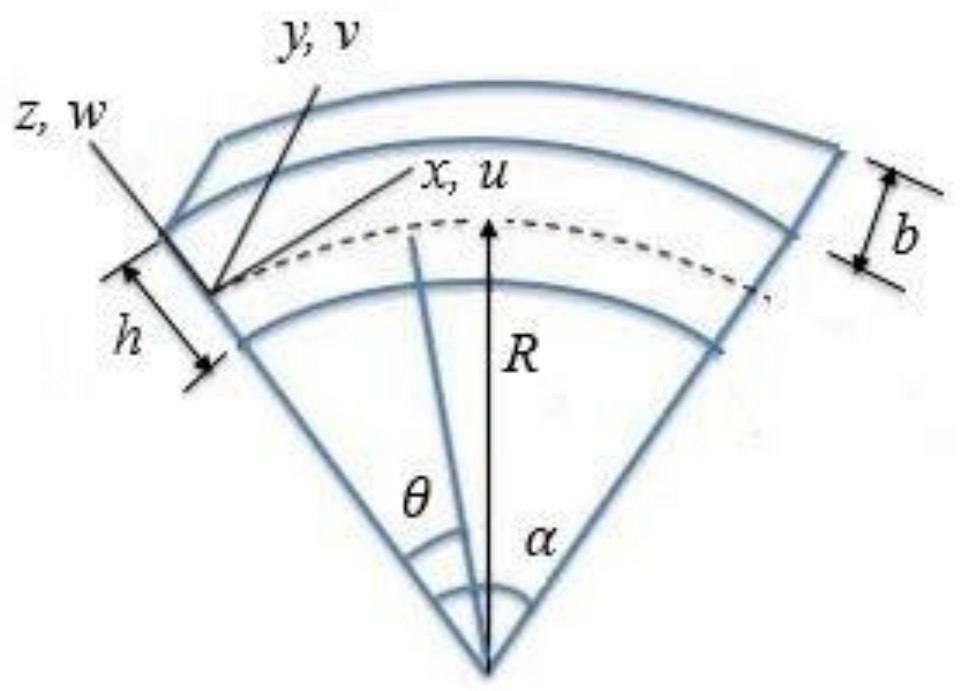

Fig. 2-1 Curved beam segment 
The midline length, $L$, of the curved beam segment is given by

$$
L=R * \alpha
$$

\subsection{Composite Lay-up Configuration}

Figure 2.1 shows a composite laminate numbered from layers 1 to $N$. The total thickness of the laminate is $h$. The $x-y$ coordinate plane is attached to the middle surface of the laminate with the positive $z$ axis up. The $z$ co-ordinate of the top of the $k^{\text {th }}$ layer is denoted as $z_{k}$ while the quantity $\overline{Z_{k}}$, represents the $z$ co-ordinate of the middle surface of the $\mathrm{k}^{\text {th }}$ layer. For a symmetric lay-up, the layers about the reference plane are mirror images of each other

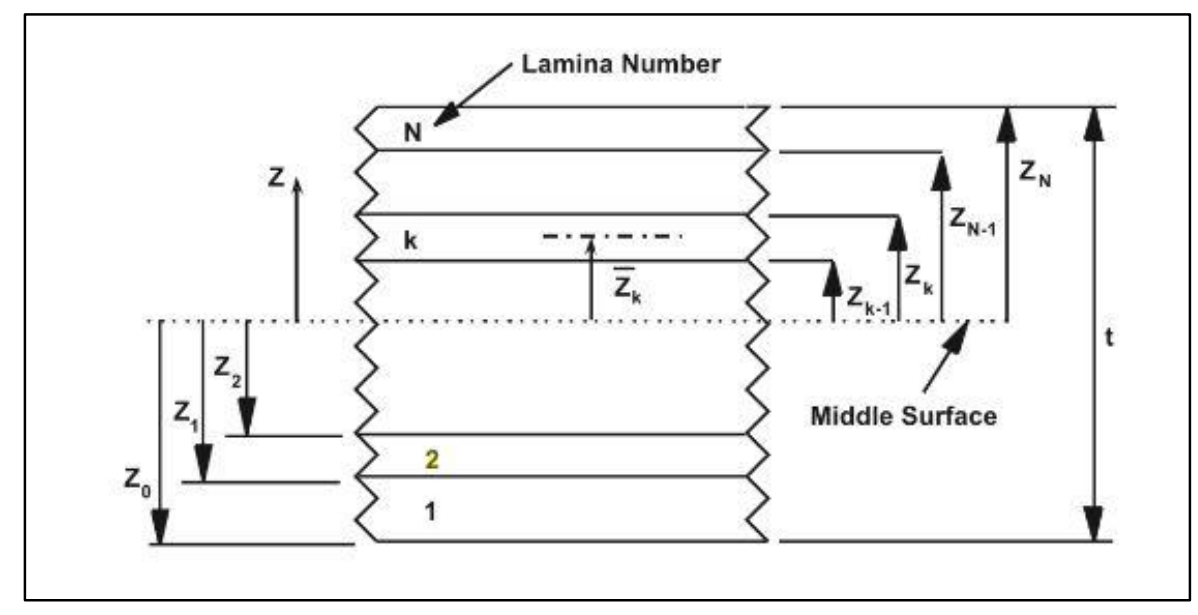

Fig 2-2 Composite Layup Configuration (Barbero, 2010)

\subsection{Plate Bending Theory}

The theory used for formulation of the curved composite beam is the Classical Laminate Plate Theory (CLPT). The classical laminate theory is a direct extension of the classical plate theory for isotropic and homogeneous material as proposed by Kirchhoff -Love. However, the extension of this theory to laminates requires some modifications to take into account the inhomogeneity in thickness direction. Kirchoff's hypotheses are used in the derivation of the 
plate stiffness and compliance equations. This theory is valid for thin plates with a side to thickness ratio greater than 10 .

The assumptions, as stated by (Reddy, 1997) for CLPT are,

(i) Straight lines perpendicular to the reference surface (i.e., transverse normal) remain straight before and after deformation.

(ii) The transverse normal does not experience elongation (i.e., they are inextensible).

(iii) The transverse normal's rotate such that they remain perpendicular to the mid-surface after deformation. ( $\varepsilon_{x z}=0$ and $\varepsilon_{y z}=0$ )

(iv) The layers are perfectly bonded together.

(v) The material of each layer is linearly elastic and has two planes of material symmetry (i.e. orthotropic)

(vi) Every layer is of uniform thickness.

(vii) The strains and displacements are small with moderate rotations.

(viii) The transverse shear stresses on the top and bottom surfaces of the laminate are zero.

\subsection{Kinematic Equations}

The kinematic equations of the curved beam segment of Fig.1 are adopted from the bending theory of cylindrical shells. Let $u, v, w$, represent the shell displacements in the $x, y, z$ directions, respectively. Further let $u_{0}$ and $v_{0}$ be the mid-surface values of $u$ and $w$, respectively; it is assumed that the transverse displacement $w$ does not vary through the thickness of the shell. 
Thus the kinematics equations for the tangential strain, $\varepsilon_{\theta}$, the lateral strain, $\varepsilon_{y}$, and the shear strain, $\gamma_{y \theta}$, are (Flugge, 1966)

$$
\begin{gathered}
\varepsilon_{\theta}=\frac{1}{R} \frac{\partial u_{o}}{\partial \theta}-\frac{z}{R(R+z)} \frac{\partial^{2} w}{\partial \theta^{2}}+\frac{w}{R+z} \\
\varepsilon_{y}=\frac{\partial v_{o}}{\partial y}-z \frac{\partial^{2} w}{\partial y^{2}} \\
\gamma_{y \theta}=\frac{1}{R+z} \frac{\partial v_{o}}{\partial \theta}+\frac{R+z}{R} \frac{\partial u_{o}}{\partial y}-\left(\frac{z}{R}+\frac{z}{R+z}\right) \frac{\partial^{2} w}{\partial y \partial \theta}
\end{gathered}
$$

Equation (2.2) can be rewritten as,

$$
\begin{gathered}
\varepsilon_{\theta}=\frac{1}{R} \frac{\partial u_{o}}{\partial \theta}-\frac{z}{R^{2}}\left(1+\frac{z}{R}\right)^{-1} \frac{\partial^{2} w}{\partial \theta^{2}}+\frac{w\left(1+\frac{z}{R}\right)^{-1}}{R} \\
\varepsilon_{y}=\frac{\partial v_{o}}{\partial y}-z \frac{\partial^{2} w}{\partial y^{2}} \\
\gamma_{y \theta}=\frac{\left(1+\frac{z}{R}\right)^{-1}}{R} \frac{\partial v_{o}}{\partial \theta}+\left(1+\frac{z}{R}\right) \frac{\partial u_{o}}{\partial y}-\frac{z}{R}\left[1+\left(1+\frac{z}{R}\right)^{-1}\right] \frac{\partial^{2} w}{\partial y \partial \theta}
\end{gathered}
$$




\section{Thin Curved Beam Equations}

A curved beam is considered thin when the ratio $h / R$ is less than 0.1 ; for a thin curved beam, we neglect the higher order terms in the binomial expansion of the term, $\left(1+\frac{z}{R}\right)^{-1}$.

$$
\left(1+\frac{Z}{R}\right)^{-1} \cong\left(1-\frac{Z}{R}\right)
$$

Then the kinematic relations become,

$$
\begin{gathered}
\varepsilon_{\theta}=\frac{1}{R} \frac{\partial u_{o}}{\partial \theta}-\frac{z\left(1-\frac{z}{R}\right)}{R^{2}} \frac{\partial^{2} w}{\partial \theta^{2}}+\frac{w\left(1-\frac{z}{R}\right)}{R} \\
\varepsilon_{y}=\frac{\partial v_{o}}{\partial y}-z \frac{\partial^{2} w}{\partial y^{2}} \\
\gamma_{y \theta}=\frac{\left(1-\frac{z}{R}\right)}{R} \frac{\partial v_{o}}{\partial \theta}+\left(1+\frac{z}{R}\right) \frac{\partial u_{o}}{\partial y}-\frac{z}{R}\left(2-\frac{z}{R}\right) \frac{\partial^{2} w}{\partial y \partial \theta}
\end{gathered}
$$

Further, neglecting $z^{2}$ terms

$$
\begin{gathered}
\varepsilon_{\theta}=\frac{1}{R} \frac{\partial u_{o}}{\partial \theta}-\frac{z}{R^{2}} \frac{\partial^{2} w}{\partial \theta^{2}}+\frac{w\left(1-\frac{z}{R}\right)}{R} \\
\varepsilon_{y}=\frac{\partial v_{o}}{\partial y}-z \frac{\partial^{2} w}{\partial y^{2}}
\end{gathered}
$$




$$
\gamma_{y \theta}=\frac{\left(1-\frac{z}{R}\right)}{R} \frac{\partial v_{o}}{\partial \theta}+\left(1+\frac{z}{R}\right) \frac{\partial u_{o}}{\partial y}-\frac{2 z}{R} \frac{\partial^{2} w}{\partial y \partial \theta}
$$

The strain components can be written as the sum of mid-plane values and that due to curvatures

as,

$$
\left\{\begin{array}{c}
\varepsilon_{\theta} \\
\varepsilon_{y} \\
\gamma_{\theta y}
\end{array}\right\}=\left\{\begin{array}{c}
\varepsilon_{\theta}^{o} \\
\varepsilon_{y}^{o} \\
\gamma_{\theta y}^{o}
\end{array}\right\}+z\left\{\begin{array}{c}
\chi_{\theta} \\
\chi_{y} \\
2 \chi_{\theta y}
\end{array}\right\}
$$

The mid-plane strains and the change in curvatures are represented as follows.

$$
\begin{gathered}
\varepsilon_{\theta}^{o}=\frac{1}{R} \frac{\partial u_{o}}{\partial \theta}+\frac{w}{R} \\
\varepsilon_{y}^{o}=\frac{\partial v_{o}}{\partial y} \\
\gamma_{y \theta}^{o}=\frac{1}{R} \frac{\partial v_{o}}{\partial \theta}+\frac{\partial u_{o}}{\partial y}=\gamma_{o} \text { (say) } \\
\chi_{\theta}=-\frac{1}{R^{2}}\left(w+\frac{\partial^{2} w}{\partial \theta^{2}}\right) \\
\chi_{y}=-\frac{\partial^{2} w}{\partial y^{2}} \\
\chi_{\theta y}=\frac{1}{R}\left(\frac{\partial u_{o}}{\partial y}-\frac{\gamma_{\theta y}^{o}}{2}-\frac{\partial^{2} w}{\partial y \partial \theta}\right)
\end{gathered}
$$




\subsection{Virtual Strain Energy}

The virtual strain energy expression for the curved beam is written as

$$
\delta U=\int_{0}^{\alpha} \iint\left(\sigma_{\theta} \delta \varepsilon_{\theta}+\sigma_{y} \delta \varepsilon_{y}+\tau_{\theta y} \delta \gamma_{\theta y}\right) d A R d \theta
$$

Substituting Eq. (2.7) in Eq. (2.10), we get,

$$
\begin{gathered}
\delta U=\int_{r_{i}}^{r_{o}} \iint\left[\sigma_{\theta}\left(\delta \varepsilon_{\theta}^{o}+z \delta \chi_{\theta}\right)+\sigma_{y}\left(\delta \varepsilon_{y}^{o}+z \delta \chi_{y}\right)+\tau_{\theta y}\left(\delta \gamma_{\theta y}^{o}\right.\right. \\
\left.+2 z \delta \chi_{\theta y}\right] d A d z
\end{gathered}
$$

The stress resultants associated with a cylindrical shell are,

$$
\begin{gathered}
N_{\theta}=\int_{-\frac{h}{2}}^{\frac{h}{2}} \sigma_{\theta} d z \\
N_{y}=\int_{-\frac{h}{2}}^{\frac{h}{2}} \sigma_{y}\left(1+\frac{z}{R}\right) d z \\
N_{\theta y}=\int_{-\frac{h}{2}}^{\frac{h}{2}} \tau_{\theta y} d z \\
N_{y \theta}=\int_{-\frac{h}{2}}^{\frac{h}{2}} \tau_{y \theta}\left(1+\frac{z}{R}\right) d z
\end{gathered}
$$




$$
\begin{gathered}
M_{\theta}=\int_{-\frac{h}{2}}^{\frac{h}{2}} \sigma_{\theta} z d z \\
M_{y}=\int_{-\frac{h}{2}}^{\frac{h}{2}} \sigma_{y}\left(1+\frac{z}{R}\right) z d z \\
M_{\theta y}=\int_{-\frac{h}{2}}^{-\frac{h}{2}} \tau_{\theta y} z d z \\
M_{y \theta}=\int_{-\frac{h}{2}}^{\frac{h}{2}} \tau_{y \theta}\left(1+\frac{z}{R}\right) z d z
\end{gathered}
$$

Eq. (2.11) can further be simplified by introducing the stress resultants from Eq. (2.12) as follows,

$$
\begin{gathered}
\delta U=b \iint\left(N_{\theta} \delta \varepsilon_{\theta}^{o}+M_{\theta} \delta \chi_{\theta}+N_{y} \delta \varepsilon_{y}^{o}+M_{y} \delta \chi_{y}+N_{\theta y} \delta \gamma_{\theta y}^{o}\right. \\
\left.+2 M_{\theta y} \delta \chi_{\theta y}\right) R d \theta
\end{gathered}
$$


For a thin curved beam, neglecting the $\frac{z}{R}$ term,

$$
N_{y \theta}=\int_{-\frac{h}{2}}^{\frac{h}{2}} \tau_{\theta y} d z \text { and } M_{y \theta}=\int_{-\frac{h}{2}}^{\frac{h}{2}} \tau_{\theta y} Z d z
$$

Thin Curved Beam $\left(\frac{\boldsymbol{h}}{\boldsymbol{R}}<\mathbf{0 . 1}\right)$ Equations with Higher Order Terms

We consider the higher order term in this section, which is shown below

$$
\left(1+\frac{Z}{R}\right)^{-1} \cong\left(1-\frac{z}{R}+\frac{z^{2}}{R^{2}}\right)
$$

Then the kinematic relations are can be written as,

$$
\begin{gathered}
\varepsilon_{\theta}=\frac{1}{R} \frac{\partial u_{o}}{\partial \theta}-\frac{z\left(1-\frac{z}{R}+\frac{z^{2}}{R^{2}}\right)}{R^{2}} \frac{\partial^{2} w}{\partial \theta^{2}}+\frac{w\left(1-\frac{z}{R}+\frac{z^{2}}{R^{2}}\right)}{R} \\
\varepsilon_{y}=\frac{\partial v_{o}}{\partial y}-z \frac{\partial^{2} w}{\partial y^{2}} \\
\gamma_{y \theta}=\frac{\left(1-\frac{z}{R}+\frac{z^{2}}{R^{2}}\right) \frac{\partial v_{o}}{\partial \theta}+\left(1+\frac{z}{R}\right) \frac{\partial u_{o}}{\partial y}-\frac{z}{R}\left(2-\frac{z}{R}+\frac{z^{2}}{R^{2}}\right) \frac{\partial^{2} w}{\partial y \partial \theta}}{R}
\end{gathered}
$$

Further, neglecting $z^{3}$ terms,

$$
\begin{gathered}
\varepsilon_{\theta}=\frac{1}{R} \frac{\partial u_{o}}{\partial \theta}-\frac{z}{R^{2}} \frac{\partial^{2} w}{\partial \theta^{2}}+\frac{z^{2}}{R^{3}} \frac{\partial^{2} w}{\partial \theta^{2}}+\frac{w\left(1-\frac{z}{R}+\frac{z^{2}}{R^{2}}\right)}{R} \\
\varepsilon_{y}=\frac{\partial v_{o}}{\partial y}-z \frac{\partial^{2} w}{\partial y^{2}}
\end{gathered}
$$




$$
\gamma_{y \theta}=\frac{\left(1-\frac{z}{R}+\frac{z^{2}}{R^{2}}\right)}{R} \frac{\partial v_{o}}{\partial \theta}+\left(1+\frac{z}{R}\right) \frac{\partial u_{o}}{\partial y}-\frac{2 z}{R} \frac{\partial^{2} w}{\partial y \partial \theta}+\frac{z^{2}}{R^{2}} \frac{\partial^{2} w}{\partial y \partial \theta}
$$

The strain that is associated with the displacement field mentioned above is as below,

$$
\left\{\begin{array}{c}
\varepsilon_{\theta} \\
\varepsilon_{y} \\
\gamma_{\theta y}
\end{array}\right\}=\left\{\begin{array}{c}
\varepsilon_{\theta}^{o} \\
\varepsilon_{y}^{o} \\
\gamma_{\theta y}^{o}
\end{array}\right\}+z\left\{\begin{array}{c}
\chi_{\theta} \\
\chi_{y} \\
2 \chi_{\theta y}
\end{array}\right\}+z^{2}\left\{\begin{array}{c}
\varepsilon_{\theta}^{2} \\
0 \\
\gamma_{\theta y}^{2}
\end{array}\right\}
$$

Rewriting the strain distributions as the sum of strain at mid-surface and that due to the change in curvatures.

$$
\begin{gathered}
\varepsilon_{\theta}=\varepsilon_{\theta}^{o}+z \chi_{\theta}+z^{2} \varepsilon_{\theta}^{(2)} \\
\varepsilon_{y}=\varepsilon_{y}^{o}+z \chi_{y} \\
\gamma_{\theta y}=\gamma_{\theta y}^{o}+z 2 \chi_{\theta y}+z^{2} \gamma_{\theta y}^{(2)}
\end{gathered}
$$

The mid-plane strains and change in curvatures are mentioned in the equation (2.8) \& (2.9), the higher order strains can be written as follows,

$$
\begin{gathered}
\varepsilon_{\theta}^{(2)}=\frac{1}{R^{3}}\left(w+\frac{\partial^{2} w}{\partial \theta^{2}}\right) \\
\gamma_{\theta y}^{(2)}=\left(\gamma_{o}-\frac{\partial u}{\partial y}\right)+\frac{1}{R^{2}} \frac{\partial^{2} w}{\partial \theta \partial y}
\end{gathered}
$$

The virtual strain energy is mentioned in equation (2.10), substituting equation (2.19) in the virtual strain energy equation, we get, 


$$
\begin{gathered}
\delta U=\iiint_{r_{i}}^{r_{o}}\left(\sigma_{\theta}\left(\delta \varepsilon_{\theta}^{o}+z \delta \chi_{\theta}+z^{2} \delta \varepsilon_{\theta}^{(2)}\right)+\sigma_{y}\left(\delta \varepsilon_{y}^{o}+z \delta \chi_{y}\right)\right. \\
+\tau_{\theta y}\left(\delta \gamma_{\theta y}^{o}+z 2 \delta \chi_{\theta y}+z^{2} \delta \gamma_{\theta y}^{(2)}\right) d A d z
\end{gathered}
$$

The stress resultants are mentioned in the equation (2.12) but for the higher order terms the stress resultants are as follows,

$$
\begin{gathered}
P_{\theta}=\int_{-\frac{h}{2}}^{\frac{h}{2}} \sigma_{\theta} z^{2} d z \\
P_{\theta y}=\int_{-\frac{h}{2}}^{\frac{h}{2}} \tau_{\theta y} z^{2} d z
\end{gathered}
$$

Equation (2.21) can further be simplified in terms of forces and moments from equation

(2.12) \& equation (2.22) as follows,

$$
\begin{gathered}
\delta U=\iint\left(N_{\theta} \delta \varepsilon_{\theta}^{o}+M_{\theta} \delta \chi_{\theta}+P_{\theta} \delta \varepsilon_{\theta}^{(2)}+N_{y} \delta \varepsilon_{y}^{o}+M_{y} \delta \chi_{y}\right. \\
\left.+N_{\theta y} \delta \gamma_{\theta y}^{o}+2 M_{\theta y} \delta \chi_{\theta y}+P_{\theta y} \delta \gamma_{\theta y}^{(2)}\right) d A
\end{gathered}
$$

\subsection{Composite Beam Formulation using Classical Laminate Plate Theory}

As seen in the literature survey in Chapter 1 , the other authors of curved beam formulation just simply set all the strain and curvature components associated with the $y$ direction to be zero. 
This practice may be passable for isotropic curved beams even though the completely neglecting the Poisson's effect is questionable; for composite beams the transverse strains and the associated elastic constants play an important role even in the case of a beam when one dimension is larger than the cross section dimensions. In the present research when reducing the shell equations to that of a curved beam, we do not set the strains and curvatures associated with the $y$ direction to be zero; instead they are eliminated using the process of static condensation.

\subsubsection{Composite Beam Formulation for Thin Curved Beam}

For a curved beam made up of composite lamina, it is assumed that the off axis of a ply follows the tangential or $\theta$ direction. For example, in a zero-degree ply the fibres follow the curvature of the beam. The constitutive equations for the CLPT are

$$
\left\{\begin{array}{c}
N_{\theta} \\
N_{y} \\
N_{\theta y} \\
M_{\theta} \\
M_{y} \\
M_{\theta y}
\end{array}\right\}=\left[\begin{array}{llllll}
A_{11} & A_{12} & A_{16} & B_{11} & B_{12} & B_{16} \\
A_{12} & A_{22} & A_{26} & B_{12} & B_{22} & B_{26} \\
A_{16} & A_{26} & A_{66} & B_{16} & B_{26} & B_{66} \\
B_{11} & B_{12} & B_{16} & D_{11} & D_{12} & D_{16} \\
B_{12} & B_{22} & B_{26} & D_{12} & D_{22} & D_{26} \\
B_{16} & B_{26} & B_{66} & D_{16} & D_{26} & D_{66}
\end{array}\right]\left\{\begin{array}{c}
\varepsilon_{\theta}^{o} \\
\varepsilon_{y}^{o} \\
\gamma_{\theta y}^{o} \\
\chi_{\theta} \\
\chi_{y} \\
2 \chi_{\theta y}
\end{array}\right\}
$$

The stress resultants $\boldsymbol{N}_{\boldsymbol{y}}$ and $\boldsymbol{M}_{\boldsymbol{y}}$ are taken to be zero to convert the plate theory to beam theory, and therefore rearranging the rows in the matrix of Eq. (2.24), 


$$
\left\{\begin{array}{c}
N_{\theta} \\
N_{\theta y} \\
M_{\theta} \\
M_{\theta y} \\
N_{y} \\
M_{y}
\end{array}\right\}=\left[\begin{array}{llllll}
A_{11} & A_{12} & A_{16} & B_{11} & B_{12} & B_{16} \\
A_{16} & A_{26} & A_{66} & B_{16} & B_{26} & B_{66} \\
B_{11} & B_{12} & B_{16} & D_{11} & D_{12} & D_{16} \\
B_{16} & B_{26} & B_{66} & D_{16} & D_{26} & D_{66} \\
A_{12} & A_{22} & A_{26} & B_{12} & B_{22} & B_{26} \\
B_{12} & B_{22} & B_{26} & D_{12} & D_{22} & D_{26}
\end{array}\right]\left\{\begin{array}{c}
\varepsilon_{\theta}^{o} \\
\varepsilon_{y}^{o} \\
\gamma_{\theta y}^{o} \\
\chi_{\theta} \\
\chi_{y} \\
2 \chi_{\theta y}
\end{array}\right\}
$$

$$
\left\{\begin{array}{c}
N_{\theta} \\
N_{\theta y} \\
M_{\theta} \\
M_{\theta y} \\
0 \\
0
\end{array}\right\}=\left[\begin{array}{llllll}
A_{11} & A_{16} & B_{11} & B_{16} & A_{12} & B_{12} \\
A_{16} & A_{66} & B_{16} & B_{66} & A_{26} & B_{26} \\
B_{11} & B_{16} & D_{11} & D_{16} & B_{12} & D_{12} \\
B_{16} & B_{66} & D_{16} & D_{66} & B_{26} & D_{26} \\
A_{12} & A_{26} & B_{12} & B_{26} & A_{22} & B_{22} \\
B_{12} & B_{26} & D_{12} & D_{26} & B_{22} & D_{22}
\end{array}\right]\left\{\begin{array}{c}
\varepsilon_{\theta}^{o} \\
\gamma_{\theta y}^{o} \\
\chi_{\theta} \\
2 \chi_{\theta y} \\
\varepsilon_{y}^{o} \\
\chi_{y}
\end{array}\right\}
$$

The partitions are named as $\left\lfloor S^{11}\right\rfloor\left\lfloor S^{12}\right\rfloor\left\lfloor S^{21}\right\rfloor \&\left[S^{22}\right\rfloor$ and thus Eq. (2.37) becomes

$$
\left\{\begin{array}{c}
N_{\theta} \\
N_{\theta y} \\
M_{\theta} \\
M_{\theta y} \\
0 \\
0
\end{array}\right\}=\left[\begin{array}{ll}
S^{11} & S^{12} \\
S^{21} & S^{22}
\end{array}\right]\left\{\begin{array}{c}
\varepsilon_{\theta}^{o} \\
\gamma_{\theta y}^{o} \\
\chi_{\theta} \\
2 \chi_{\theta y} \\
\varepsilon_{y}^{o} \\
\chi_{y}
\end{array}\right\}
$$

Equation (2.27) can be written as,

$$
\left[\begin{array}{llll}
N_{\theta} & N_{\theta y} & M_{\theta} & M_{\theta y}
\end{array}\right]^{T}=[S]\left[\begin{array}{llll}
\varepsilon_{\theta} & \gamma_{\theta y} & \chi_{\theta} & 2 \chi_{\theta y}
\end{array}\right]^{T}
$$

where the $[S]$ matrix is defined as

$$
[S]=\left[S^{11}\right]-\left[S^{12}\right]\left[S^{22}\right]^{-1}\left[S^{21}\right]
$$




$$
\begin{gathered}
{[S]=\left[\begin{array}{llll}
S_{11} & S_{12} & S_{13} & S_{14} \\
S_{21} & S_{22} & S_{23} & S_{24} \\
S_{31} & S_{32} & S_{33} & S_{34} \\
S_{41} & S_{42} & S_{43} & S_{44}
\end{array}\right]} \\
\left.\left\{\begin{array}{c}
N_{\theta} \\
N_{\theta y} \\
M_{\theta} \\
M_{\theta y}
\end{array}\right\}=\left[\begin{array}{llll}
S_{11} & S_{12} & S_{13} & S_{14} \\
S_{21} & S_{22} & S_{23} & S_{24} \\
S_{31} & S_{32} & S_{33} & S_{34} \\
S_{41} & S_{42} & S_{43} & S_{44}
\end{array}\right]\left\{\begin{array}{c}
\frac{1}{R} u^{\theta}+\frac{w}{R} \\
\gamma_{o} \\
-\frac{1}{R^{2}}\left(w+w^{\theta \theta}\right) \\
2\left[\frac{1}{R}\left(u^{y}-\frac{\gamma_{o}}{2}-w^{y \theta}\right)\right.
\end{array}\right]\right) \\
N_{\theta}=\left(\frac{S_{11}}{R}\right) u^{\theta}+\left(S_{12}-\frac{S_{14}}{2 R}\right) \gamma_{o}+\left(\frac{S_{11}}{R}-\frac{S_{13}}{R^{2}}\right) w-\frac{S_{13}}{R^{2}} w^{\theta \theta} \\
+2 \frac{S_{14}}{R} u^{y}-2 \frac{S_{14}}{R} w^{y \theta}
\end{gathered}
$$

$$
\begin{gathered}
N_{\theta y}=\left(\frac{S_{21}}{R}\right) u^{\theta}+\left(S_{22}-\frac{S_{24}}{2 R}\right) \gamma_{o}+\left(\frac{S_{21}}{R}-\frac{S_{23}}{R^{2}}\right) w-\frac{S_{23}}{R^{2}} w^{\theta \theta} \\
+2 \frac{S_{24}}{R} u^{y}-2 \frac{S_{24}}{R} w^{y \theta}
\end{gathered}
$$

$$
\begin{aligned}
M_{\theta}=\left(\frac{S_{31}}{R}\right) & u^{\theta}+\left(S_{32}-\frac{S_{34}}{2 R}\right) \gamma_{o}+\left(\frac{S_{31}}{R}-\frac{S_{33}}{R^{2}}\right) w-\frac{S_{33}}{R^{2}} w^{\theta \theta} \\
& +2 \frac{S_{34}}{R} u^{y}-2 \frac{S_{34}}{R} w^{y \theta}
\end{aligned}
$$




$$
\begin{gathered}
M_{\theta y}=\left(\frac{S_{41}}{R}\right) u^{\theta}+\left(S_{42}-\frac{S_{44}}{2 R}\right) \gamma_{o}+\left(\frac{S_{41}}{R}-\frac{S_{43}}{R^{2}}\right) w-\frac{S_{43}}{R^{2}} w^{\theta \theta} \\
+2 \frac{S_{44}}{R} u^{y}-2 \frac{S_{44}}{R} w^{y \theta}
\end{gathered}
$$

The equation (2.32) above represent the stress and moment resultants derived from equation (2.31).

$u=$ axial deformation at the mid - plane

$u^{y}=$ differential of $u$ along the transverse direction

$\gamma=$ mid - plane shear strain

$w=$ lateral deformation

$\mathrm{w}^{\prime}=$ slope

$w^{y}=$ twist angle associated to lateral deformation

Equation (2.23) is reproduced here for convenience:

$$
\delta U=R b \int_{0}^{\alpha}\left(N_{\theta} \delta \varepsilon_{\theta}^{o}+M_{\theta} \delta \chi_{\theta}+N_{\theta y} \delta \gamma_{\theta y}^{o}+2 M_{\theta y} \delta \chi_{\theta y}\right) d \theta
$$

where the virtual strains can be written as,

$$
\delta \varepsilon_{\theta}^{o}=\frac{1}{R} \frac{\partial \delta u_{o}}{\partial \theta}+\frac{\delta w}{R}=\frac{1}{R}\left(\delta u^{\theta}+\delta w\right)
$$




$$
\begin{gathered}
\delta \gamma_{y \theta}^{o}=\frac{1}{R} \frac{\partial \delta v_{o}}{\partial \theta}+\frac{\partial \delta u_{o}}{\partial y}=\delta \gamma_{o} \\
\delta \chi_{\theta}=-\frac{1}{R^{2}}\left(\delta w+\frac{\partial^{2} \delta w}{\partial \theta^{2}}\right)=-\frac{1}{R^{2}}\left(\delta w^{\theta \theta}+\delta w\right) \\
\delta \chi_{\theta y}=\frac{1}{R}\left(\frac{\partial \delta u_{o}}{\partial y}-\frac{\delta \gamma_{\theta y}^{o}}{2}-\frac{\partial^{2} \delta w}{\partial y \partial \theta}\right)=\frac{1}{R}\left(\delta u^{y}-\frac{\delta \gamma_{o}}{2}-\delta w^{y \theta}\right)
\end{gathered}
$$

Substituting equation (2.9) in equation (2.33)

$$
\begin{array}{r}
\delta U=R b \int_{0}^{\alpha}\left(N_{\theta} \frac{1}{R}\left(\delta u^{\theta}+\delta w\right)-M_{\theta}\left(\frac{1}{R^{2}}\left(\delta w^{\theta \theta}+\delta w\right)\right)\right. \\
\left.+N_{\theta y} \delta \gamma_{o}+2 M_{\theta y} \frac{1}{R}\left(\delta u^{y}-\frac{\delta \gamma_{o}}{2}-\delta w^{y \theta}\right)\right) d \theta
\end{array}
$$

\subsubsection{Composite Beam Formulation for Higher Order Term}

This section deals with the CLPT formulation which explains Plane Stress Case. The constitutive equation is given below and is represented in matrix form. 


$$
\left\{\begin{array}{c}
N_{\theta} \\
N_{y} \\
N_{\theta y} \\
M_{\theta} \\
M_{y} \\
M_{\theta y} \\
P_{\theta} \\
P_{y} \\
P_{\theta y}
\end{array}\right\}=\left[\begin{array}{lllllllll}
A_{11} & A_{12} & A_{16} & B_{11} & B_{12} & B_{16} & E_{11} & E_{12} & E_{16} \\
A_{21} & A_{22} & A_{26} & B_{21} & B_{22} & B_{26} & E_{21} & E_{22} & E_{26} \\
A_{61} & A_{62} & A_{66} & B_{61} & B_{62} & B_{66} & E_{61} & E_{62} & E_{66} \\
B_{11} & B_{12} & B_{16} & D_{11} & D_{12} & D_{16} & F_{11} & F_{12} & F_{16} \\
B_{21} & B_{22} & B_{26} & D_{21} & D_{22} & D_{26} & F_{21} & F_{22} & F_{26} \\
B_{61} & B_{62} & B_{66} & D_{61} & D_{62} & D_{66} & F_{61} & F_{62} & F_{66} \\
E_{11} & E_{12} & E_{16} & F_{11} & F_{12} & F_{16} & H_{11} & H_{12} & H_{16} \\
E_{21} & E_{22} & E_{26} & F_{21} & F_{22} & F_{26} & H_{21} & H_{22} & H_{26} \\
E_{61} & E_{62} & E_{66} & F_{61} & F_{62} & F_{66} & H_{61} & H_{62} & H_{66}
\end{array}\right]\left\{\begin{array}{c}
\varepsilon_{\theta}^{o} \\
\varepsilon_{y}^{o} \\
\gamma_{\theta y}^{o} \\
\chi_{\theta} \\
\chi_{y} \\
2 \chi_{\theta y} \\
\varepsilon_{\theta}^{(2)} \\
0 \\
\gamma_{\theta y}^{(2)}
\end{array}\right\}
$$

By setting $N_{y}, M_{y} \& P_{y}$ to be zeros. The plate theory is converted to beam theory, and therefore arranging the terms in the matrix as per equation (2.36)

$$
\left\{\begin{array}{c}
N_{\theta} \\
N_{\theta y} \\
M_{\theta} \\
M_{\theta y} \\
P_{\theta} \\
P_{\theta y} \\
0 \\
0 \\
0
\end{array}\right\}=\left[\begin{array}{llllll|lll}
A_{11} & A_{16} & B_{11} & B_{16} & E_{11} & E_{16} & A_{12} & B_{12} & E_{12} \\
A_{16} & A_{66} & B_{16} & B_{66} & E_{16} & E_{66} & A_{26} & B_{26} & E_{26} \\
B_{11} & B_{16} & D_{11} & D_{16} & F_{11} & F_{16} & B_{12} & D_{12} & F_{12} \\
B_{16} & B_{66} & D_{16} & D_{66} & F_{16} & F_{66} & B_{26} & D_{26} & F_{26} \\
E_{11} & E_{16} & F_{11} & F_{16} & H_{11} & H_{16} & E_{12} & F_{12} & H_{12} \\
E_{16} & E_{66} & F_{16} & F_{66} & H_{16} & H_{66} & E_{26} & F_{26} & H_{26} \\
A_{12} & A_{26} & B_{12} & B_{26} & E_{12} & E_{26} & A_{22} & B_{22} & E_{22} \\
B_{12} & B_{26} & D_{12} & D_{26} & F_{12} & F_{26} & B_{22} & D_{22} & F_{22} \\
E_{12} & E_{26} & F_{12} & F_{26} & H_{12} & H_{26} & E_{22} & F_{22} & H_{22}
\end{array}\right]\left\{\begin{array}{c}
\varepsilon_{\theta}^{o} \\
\gamma_{\theta y}^{o} \\
\chi_{\theta} \\
2 \chi_{\theta y} \\
\varepsilon_{\theta}^{(2)} \\
\gamma_{\theta y}^{(2)} \\
\varepsilon_{y}^{o} \\
\chi_{y} \\
0
\end{array}\right\}
$$

The partitions are named as $\left\lfloor T^{11}\right\rfloor\left\lfloor T^{12}\right\rfloor\left\lfloor T^{21}\right\rfloor \&\left\lfloor T^{22}\right\rfloor$ and represented as follows. 


$$
\left\{\begin{array}{c}
N_{\theta} \\
N_{\theta y} \\
M_{\theta} \\
M_{\theta y} \\
P_{\theta} \\
P_{\theta y} \\
0 \\
0 \\
0
\end{array}\right\}=\left[\begin{array}{ll}
T^{11} & T^{12} \\
T^{21} & T^{22}
\end{array}\right]\left\{\begin{array}{c}
\varepsilon_{\theta}^{o} \\
\gamma_{\theta y}^{o} \\
\chi_{\theta} \\
2 \chi_{\theta y} \\
\varepsilon_{\theta}^{(2)} \\
\gamma_{\theta y}^{(2)} \\
\varepsilon_{y}^{o} \\
\chi_{y} \\
0
\end{array}\right\}
$$

Therefore, the equation (2.38) can be written as below,

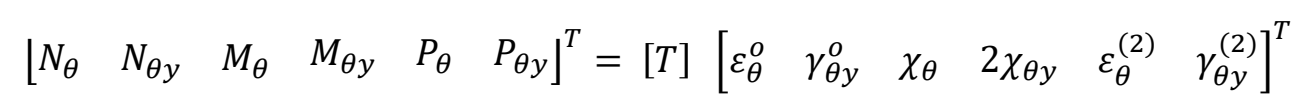

Where $[T]$ can be expressed as follow,

$$
\begin{gathered}
{[T]=\left[T^{11}\right]-\left[T^{12}\right]\left[T^{22}\right]^{-1}\left[T^{21}\right]} \\
{[T]=\left[\begin{array}{lllllll}
T_{11} & T_{12} & T_{13} & T_{14} & T_{15} & T_{16} \\
T_{21} & T_{22} & T_{23} & T_{24} & T_{25} & T_{26} \\
T_{31} & T_{32} & T_{33} & T_{34} & T_{35} & T_{36} \\
T_{41} & T_{42} & T_{43} & T_{44} & T_{45} & T_{46} \\
T_{51} & T_{52} & T_{53} & T_{54} & T_{55} & T_{56} \\
T_{61} & T_{62} & T_{63} & T_{64} & T_{65} & T_{66}
\end{array}\right]}
\end{gathered}
$$




$$
\left\{\begin{array}{c}
N_{\theta} \\
N_{\theta y} \\
M_{\theta} \\
M_{\theta y} \\
P_{\theta} \\
P_{\theta y}
\end{array}\right\}=\left[\begin{array}{llllll}
T_{11} & T_{12} & T_{13} & T_{14} & T_{15} & T_{16} \\
T_{21} & T_{22} & T_{23} & T_{24} & T_{25} & T_{26} \\
T_{31} & T_{32} & T_{33} & T_{34} & T_{35} & T_{36} \\
T_{41} & T_{42} & T_{43} & T_{44} & T_{45} & T_{46} \\
T_{51} & T_{52} & T_{53} & T_{54} & T_{55} & T_{56} \\
T_{61} & T_{62} & T_{63} & T_{64} & T_{65} & T_{66}
\end{array}\right]\left\{\begin{array}{c}
\frac{1}{R} u^{\theta}+\frac{w}{R} \\
\gamma_{o} \\
-\frac{1}{R^{2}}\left(w+w^{\theta \theta}\right) \\
2\left(\frac{1}{R}\left(u^{y}-\frac{\gamma_{o}}{2}-w^{y \theta}\right)\right. \\
\frac{1}{R^{3}}\left(w+w^{\theta \theta}\right) \\
\frac{1}{R^{2}}\left(-u^{y}+\gamma_{o}+w^{y \theta}\right)
\end{array}\right\}
$$

Where the forces, moments and higher order terms are written as follows,

$$
\begin{aligned}
& N_{\theta}=\left(\frac{T_{11}}{R}\right) u^{\theta}+\left(\frac{2 T_{14}}{R}-\frac{T_{16}}{R^{2}}\right) u^{y}+\left(T_{12}-\frac{T_{14}}{R}+\frac{T_{16}}{R^{2}}\right) \gamma_{o} \\
& +\left(-\frac{T_{13}}{R^{2}}+\frac{T_{15}}{R^{3}}+\frac{T_{11}}{R}\right) w+\left(-\frac{T_{13}}{R^{2}}+\frac{T_{15}}{R^{3}}\right) w^{\theta \theta} \\
& +\left(-\frac{2 T_{14}}{R}+\frac{T_{16}}{R^{2}}\right) w^{y \theta} \\
& N_{\theta y}=\left(\frac{T_{21}}{R}\right) u^{\theta}+\left(\frac{2 T_{24}}{R}-\frac{T_{26}}{R^{2}}\right) u^{y}+\left(T_{22}-\frac{T_{24}}{R}+\frac{T_{26}}{R^{2}}\right) \gamma_{o} \\
& +\left(-\frac{T_{23}}{R^{2}}+\frac{T_{25}}{R^{3}}+\frac{T_{21}}{R}\right) w+\left(-\frac{T_{23}}{R^{2}}+\frac{T_{25}}{R^{3}}\right) w^{\theta \theta} \\
& +\left(-\frac{2 T_{24}}{R}+\frac{T_{26}}{R^{2}}\right) w^{y \theta} \\
& M_{\theta}=\left(\frac{T_{31}}{R}\right) u^{\theta}+\left(\frac{2 T_{34}}{R}-\frac{T_{36}}{R^{2}}\right) u^{y}+\left(T_{32}-\frac{T_{34}}{R}+\frac{T_{36}}{R^{2}}\right) \gamma_{o} \\
& +\left(-\frac{T_{33}}{R^{2}}+\frac{T_{35}}{R^{3}}+\frac{T_{31}}{R}\right) w+\left(-\frac{T_{33}}{R^{2}}+\frac{T_{35}}{R^{3}}\right) w^{\theta \theta} \\
& +\left(-\frac{2 T_{34}}{R}+\frac{T_{36}}{R^{2}}\right) w^{y \theta}
\end{aligned}
$$




$$
\begin{aligned}
& M_{\theta y}=\left(\frac{T_{41}}{R}\right) u^{\theta}+\left(\frac{2 T_{44}}{R}-\frac{T_{46}}{R^{2}}\right) u^{y}+\left(T_{42}-\frac{T_{44}}{R}+\frac{T_{46}}{R^{2}}\right) \gamma_{o} \\
& +\left(-\frac{T_{43}}{R^{2}}+\frac{T_{45}}{R^{3}}+\frac{T_{41}}{R}\right) w+\left(-\frac{T_{43}}{R^{2}}+\frac{T_{45}}{R^{3}}\right) w^{\theta \theta} \\
& +\left(-\frac{2 T_{44}}{R}+\frac{T_{46}}{R^{2}}\right) w^{y \theta} \\
& P_{\theta}=\left(\frac{T_{51}}{R}\right) u^{\theta}+\left(\frac{2 T_{54}}{R}-\frac{T_{56}}{R^{2}}\right) u^{y}+\left(T_{52}-\frac{T_{54}}{R}+\frac{T_{56}}{R^{2}}\right) \gamma_{o} \\
& +\left(-\frac{T_{53}}{R^{2}}+\frac{T_{55}}{R^{3}}+\frac{T_{51}}{R}\right) w+\left(-\frac{T_{53}}{R^{2}}+\frac{T_{55}}{R^{3}}\right) w^{\theta \theta} \\
& +\left(-\frac{2 T_{54}}{R}+\frac{T_{56}}{R^{2}}\right) w^{y \theta} \\
& P_{\theta y}=\left(\frac{T_{61}}{R}\right) u^{\theta}+\left(\frac{2 T_{64}}{R}-\frac{T_{66}}{R^{2}}\right) u^{y}+\left(T_{62}-\frac{T_{64}}{R}+\frac{T_{66}}{R^{2}}\right) \gamma_{o} \\
& +\left(-\frac{T_{63}}{R^{2}}+\frac{T_{65}}{R^{3}}+\frac{T_{61}}{R}\right) w+\left(-\frac{T_{63}}{R^{2}}+\frac{T_{65}}{R^{3}}\right) w^{\theta \theta} \\
& +\left(-\frac{2 T_{64}}{R}+\frac{T_{66}}{R^{2}}\right) w^{y \theta}
\end{aligned}
$$

Equation (2.23) can now be further simplified as per plane stress case as below.

$$
\begin{gathered}
\delta U=\iint\left(N_{\theta} \delta \varepsilon_{\theta}^{o}+M_{\theta} \delta \chi_{\theta}+P_{\theta} \delta \varepsilon_{\theta}^{(2)}+N_{\theta y} \delta \gamma_{\theta y}^{o}+2 M_{\theta y} \delta \chi_{\theta y}\right. \\
\left.+P_{\theta y} \delta \gamma_{\theta y}^{(2)}\right) d A
\end{gathered}
$$


The area integral for equation (2.44) can now be written as,

$$
\begin{gathered}
\delta U=R b \int_{0}^{\alpha}\left(N_{\theta} \delta \varepsilon_{\theta}^{o}+M_{\theta} \delta \chi_{\theta}+P_{\theta} \delta \varepsilon_{\theta}^{(2)}+N_{\theta y} \delta \gamma_{\theta y}^{o}\right. \\
\left.+2 M_{\theta y} \delta \chi_{\theta y}+P_{\theta y} \delta \gamma_{\theta y}^{(2)}\right) d \theta
\end{gathered}
$$

Where the strain derivatives can be written as follows,

$$
\begin{aligned}
& \delta \varepsilon_{\theta}^{o}=\frac{1}{R} \frac{\partial \delta u_{o}}{\partial \theta}+\frac{\delta w}{R}=\frac{1}{R}\left(\delta u^{\theta}+\delta w\right) \\
& \delta \gamma_{y \theta}^{o}=\frac{1}{R} \frac{\partial \delta v_{o}}{\partial \theta}+\frac{\partial \delta u_{o}}{\partial y}=\delta \gamma_{o} \\
& \delta \chi_{\theta}=-\frac{1}{R^{2}}\left(\delta w+\frac{\partial^{2} \delta w}{\partial \theta^{2}}\right)=-\frac{1}{R^{2}}\left(\delta w^{\theta \theta}+\delta w\right) \\
& \delta \chi_{\theta y}=\frac{1}{R}\left(\frac{\partial \delta u_{o}}{\partial y}-\frac{\delta \gamma_{\theta y}^{o}}{2}-\frac{\partial^{2} \delta w}{\partial y \partial \theta}\right)=\frac{1}{R}\left(\delta u^{y}-\frac{\delta \gamma_{o}}{2}-\delta w^{y \theta}\right) \\
& \delta \varepsilon_{\theta}^{(2)}=\frac{1}{R^{3}}\left(\delta w+\frac{\partial^{2} \delta w}{\partial \theta^{2}}\right)=\frac{1}{R^{3}}\left(\delta w^{\theta \theta}+\delta w\right) \\
& \delta \gamma_{y \theta}^{(2)} \frac{1}{R^{2}}\left(-\frac{\partial \delta u_{o}}{\partial y}+\delta \gamma_{\theta y}^{o}+\frac{\partial^{2} \delta w}{\partial y \partial \theta}\right)=\frac{1}{R^{2}}\left(-\delta u^{y}+\delta \gamma_{o}+\delta w^{y \theta}\right)
\end{aligned}
$$

Substituting equation (2.46) in equation (2.45) 


$$
\begin{aligned}
\delta U=R b \int_{0}^{\alpha}( & \left(\frac{N_{\theta}}{R}\left(\delta u^{\theta}+\delta w\right)\right)-\left(\frac{M_{\theta}}{R^{2}}\left(\delta w^{\theta \theta}+\delta w\right)\right) \\
& +\left(\frac{P_{\theta}}{R^{3}}\left(\delta w^{\theta \theta}+\delta w\right)\right)+N_{\theta y} \delta \gamma_{o} \\
& +\left(\frac{2 M_{\theta y}}{R}\left(\delta u^{y}-\frac{\delta \gamma_{o}}{2}-\delta w^{y \theta}\right)\right) \\
& \left.+\left(\frac{P_{\theta y}}{R^{2}}\left(-\delta u^{y}+\delta \gamma_{o}+\delta w^{y \theta}\right)\right)\right) d \theta_{e}
\end{aligned}
$$




\section{FINITE ELEMENT FORMULATION}

\subsection{Introduction}

Partial differential equations are a mathematical way to represent many engineering problems, but this has its limitations, since it is not possible to obtain solutions in closed form in all engineering cases; therefore, we employ numerical methods in such cases to arrive at approximate solutions. The closed form solution in a domain is valid at all points but numerical methods give approximate solution only at certain discrete points in the domain. As we increase the number of elements or the number of internal nodes in an element or both we can approach the exact solution. In this chapter the procedure and quantities associated with the finite element formulation are detailed. Further the step-by-step procedure for determining the strain and stress fields at a given cross section is outlined.

\subsection{Finite Element Methods}

The three different types of finite element methods are the $h$ version, $p$ version and $h-p$ version. In the $h$-version if the number of elements that are used to discretize the domain are increased the accuracy of the results is improved. But for better accuracy one can use the $p$ version where the order of the shape function is increased by introducing more internal nodes. The $h-p$ version is a combination of the $h$ version and the $p$ version wherein both the number of elements in the model and the number of internal nodes are increased. The $h-p$ version is used in this research. The Lagrange and Hermite polynomials are used to define the shape functions. 
As per the convergence study conducted by (Sreeram \& Sivaneri, 1997) it is suggested that beams with three internal nodes are sufficient for the analysis.

\subsection{Finite Element Shape Functions}

In the present finite element model, a typical finite element contains two end nodes and three internal nodes. If a dependent variable of a finite element is forced to have continuity of only the variable at element junctions during the process of assembly, it is said to possess $\mathrm{C}^{0}$ continuity; it is $\mathrm{C}^{1}$ continuity if the continuity of the slope also is assured at an element junction. The shape functions for a variable with $\mathrm{C}^{0}$ continuity are derived from Lagrange polynomials while that for the ones with $\mathrm{C}^{1}$ continuity are derived from Hermite polynomials for the variables that satisfy $C^{1}$ continuity, slope degrees of freedom are assumed only at the end nodes since the slope continuity at the internal nodes is assured automatically.

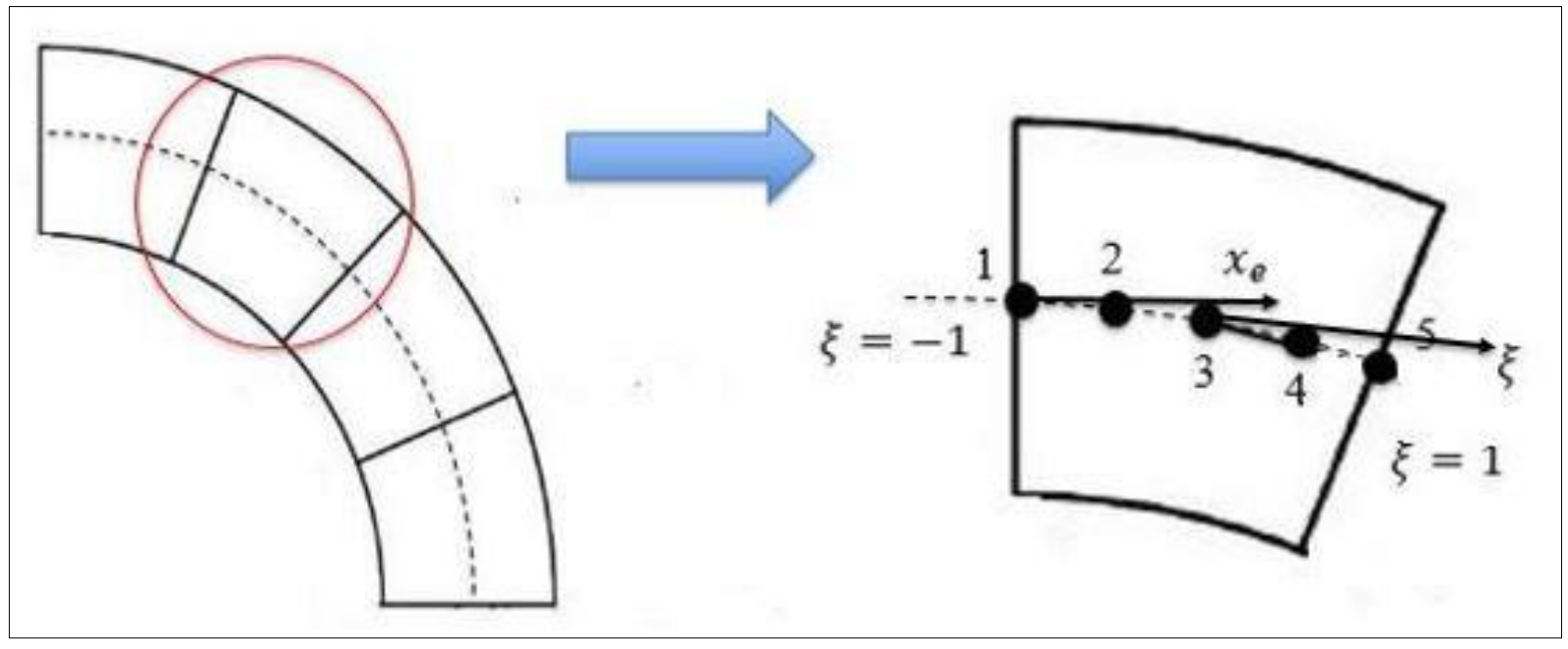

Fig 3-1: Element definition for reduced beam formulation using CLPT

Consider a finite element in the form of a circular arc of radius $R$ as shown in Fig. 3.1. The local co-ordinate $\vartheta_{e}$ is fixed to the left end of the element and ranges from 0 to $\alpha_{e}$, where $\alpha_{e}$ is the 
angular extent of the element. The mid surface curved length of the element is $l_{e}$. The elemental coordinate $x_{e}$ is measured from the left end of the element along the tangent to the midline of the element. The non-dimensional coordinate $\xi$ is attached to the center of the element, i.e., at node 3 and it ranges from -1 to +1 . As seen in Fig. 3.1, the dependent variables at the nodes are $u, \gamma, w, u_{y}, w_{y}$ of which $u, \gamma, u_{y}, w_{y}$ obey $C^{0}$ continuity and $w$ obeys $C^{1}$ continuity. The relationship between the curved length and angular length of the element is given by

$$
l_{e}=R \alpha_{e}
$$

The coordinate transformation between $x_{e}$ and $\xi$ is given by

$$
\begin{gathered}
x_{e}=\frac{l_{e}}{2}(1+\xi) \\
d x_{e}=\frac{l_{e}}{2} d \xi
\end{gathered}
$$

The distribution for $u(\xi)$, the axial degrees of freedom is expressed as,

$$
u(\xi)=\sum_{i=0}^{4} a_{i} \xi^{i}
$$

The distribution $\mathrm{w}(\xi)$ for the transverse degrees of freedom is assumed as,

$$
w(\xi)=\sum_{j=0}^{6} b_{j} \xi^{j}
$$

where $a_{i}$ and $b_{j}$ are generalized coordinates that are to be determined. In general, these equations can be written in matrix notations as 


$$
\begin{aligned}
& u(\xi)=\left\lfloor\xi^{i}\right\rfloor\left\{a_{i}\right\} \\
& w(\xi)=\left\lfloor\xi^{j}\right\rfloor\left\{b_{j}\right\}
\end{aligned}
$$

To solve for the ai's in Eq. (3.6), five equations are required and the axial degrees of freedom at the nodes of the element are considered.

$$
\begin{gathered}
u(-1)=u_{1} \\
u(-0.5)=u_{2} \\
u(0)=u_{3} \\
u(0.5)=u_{4} \\
u(1)=u_{5}
\end{gathered}
$$

On solving the above equations, Eq. (3.6) can be rewritten in terms of Lagrange polynomials as

$$
u(\xi)=\left\lfloor\begin{array}{lll}
H_{L 1} & \cdots & H_{L 5}
\end{array}\right\rfloor\left\{\begin{array}{c}
u_{1} \\
\vdots \\
u_{5}
\end{array}\right\}
$$

where $H_{L 1}(\xi), H_{L 2}(\xi)$, etc., are called Lagrangian shape functions and are given by,

$$
\begin{gathered}
H_{L 1}=\frac{1}{6} \xi-\frac{1}{6} \xi^{2}-\frac{2}{3} \xi^{3}+\frac{2}{3} \xi^{4} \\
H_{L 2}=-\frac{4}{3} \xi+\frac{8}{3} \xi^{2}+\frac{4}{3} \xi^{3}-\frac{8}{3} \xi^{4} \\
H_{L 3}=1-5 \xi^{2}+4 \xi^{4}
\end{gathered}
$$




$$
\begin{gathered}
H_{L 4}=\frac{4}{3} \xi+\frac{8}{3} \xi^{2}-\frac{4}{3} \xi^{3}-\frac{8}{3} \xi^{4} \\
H_{L 5}=-\frac{1}{6} \xi-\frac{1}{6} \xi^{2}+\frac{2}{3} \xi^{3}+\frac{2}{3} \xi^{4}
\end{gathered}
$$

Similarly, to solve for the $b_{j}^{\prime}$ s in Eq. (3.7) seven equations are required and the transverse degrees of freedom at the nodes of an element are considered.

$$
\begin{gathered}
w(-1)=w_{1} \\
\frac{l_{e}}{2} w^{\prime}(-1)=w_{1}{ }^{\prime} \\
w(-0.5)=w_{2} \\
w(0)=w_{3} \\
w(0.5)=w_{4} \\
w(1)=w_{5} \\
\frac{l_{e}}{2} w^{\prime}(1)=w_{5}{ }^{\prime}
\end{gathered}
$$

On solving the above equations, (3.7) can be rewritten in terms of Hermite polynomials:

$$
w(\xi)=\left\lfloor\begin{array}{lll}
H_{1} & \cdots & H_{5}
\end{array}\right\rfloor\left\{\begin{array}{c}
w_{1} \\
w_{1}{ }^{\prime} \\
\vdots \\
w_{5} \\
w_{5}{ }^{\prime}
\end{array}\right\}
$$

where $H_{1}(\xi), H_{2}(\xi)$ etc., are called Hermite shape functions and are given by 


$$
\begin{gathered}
H_{1}=\frac{1}{9}\left(\frac{17}{4} \xi-5 \xi^{2}-\frac{79}{4} \xi^{3}+\frac{47}{2} \xi^{4}+11 \xi^{5}-14 \xi^{6}\right) \\
H_{2}=\frac{l_{e}}{6}\left(\frac{1}{4} \xi-\frac{1}{4} \xi^{2}-\frac{5}{4} \xi^{3}+\frac{5}{4} \xi^{4}+\xi^{5}-\xi^{6}\right) \\
H_{3}=\frac{16}{9}\left(-\xi+2 \xi^{2}+2 \xi^{3}-4 \xi^{4}-\xi^{5}+2 \xi^{6}\right) \\
H_{5}=\frac{16}{9}\left(\xi+2 \xi^{2}-2 \xi^{3}-4 \xi^{4}+\xi^{5}+2 \xi^{6}\right) \\
H_{6}=\frac{1}{9}\left(-\frac{17}{4} \xi-5 \xi^{2}+\frac{79}{4} \xi^{3}+\frac{47}{2} \xi^{4}-11 \xi^{5}-14 \xi^{6}\right) \\
H_{7}=\frac{l_{e}}{6}\left(\frac{1}{4} \xi+\frac{1}{4} \xi^{2}-\frac{5}{4} \xi^{3}-\frac{5}{4} \xi^{4}+\xi^{5}+\xi^{6}\right)
\end{gathered}
$$

\subsection{Element Stiffness Formulation using CLPT}

The number of degrees of freedom of a node are different for end nodes and internal nodes. At end nodes the degrees of freedom are: $u, \gamma, w, w^{\prime}, u_{y}, w_{y}$ while at internal nodes the degrees of freedom are: $u, \gamma, w, u_{y}, w_{y}$. The total degrees of freedom for the element are 27 .

$$
\begin{aligned}
& u=\left\lfloor H_{L}\right\rfloor\left\{q_{u}\right\} \\
& \gamma=\left\lfloor H_{L}\right\rfloor\left\{q_{\gamma}\right\}
\end{aligned}
$$




$$
\begin{gathered}
w=\lfloor H\rfloor\left\{q_{w}\right\} \\
u_{y}=\left\lfloor H_{L}\right\rfloor\left\{q_{u_{y}}\right\} \\
w_{y}=\left\lfloor H_{L}\right\rfloor\left\{q_{w_{y}}\right\}
\end{gathered}
$$

The stiffness matrix is obtained from the virtual strain energy expression [Eq. (2.33)]. The virtual forms of the dependent variables are,

$$
\begin{gathered}
\delta u=\left\lfloor\delta q_{u}\right\rfloor\left\{H_{L}\right\} \\
\delta \gamma=\left\lfloor\delta q_{\gamma}\right\rfloor\left\{H_{L}\right\} \\
\delta w=\left\lfloor\delta q_{w}\right\rfloor\{H\} \\
\delta u_{y}=\left\lfloor\delta q_{u_{y}}\right\rfloor\left\{H_{L}\right\} \\
\delta w_{y}=\left\lfloor\delta q_{w_{y}}\right\rfloor\left\{H_{L}\right\}
\end{gathered}
$$

where,

$$
\begin{aligned}
& \left\lfloor q_{u}\right\rfloor=\left\lfloor\begin{array}{lllll}
u_{1} & u_{2} & u_{3} & u_{4} & u_{5}
\end{array}\right\rfloor \\
& \left\lfloor\delta q_{u}\right\rfloor=\left\lfloor\delta u_{1} \delta u_{2} \delta u_{3} \delta u_{4} \delta u_{5}\right\rfloor \\
& \left\lfloor q_{\gamma}\right\rfloor=\left\lfloor\gamma_{1} \gamma_{2} \gamma_{3} \gamma_{4} \gamma_{5}\right\rfloor \\
& \left\lfloor\delta q_{\gamma}\right\rfloor=\left\lfloor\delta \gamma_{1} \delta \gamma_{2} \delta \gamma_{3} \delta \gamma_{4} \delta \gamma_{5}\right\rfloor
\end{aligned}
$$

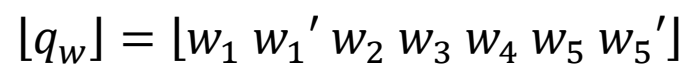




$$
\begin{gathered}
\left\lfloor\delta q_{w}\right\rfloor=\left\lfloor\delta w_{1} \delta w_{1}^{\prime} \delta w_{2} \delta w_{3} \delta w_{4} \delta w_{5} \delta w_{5}{ }^{\prime}\right\rfloor \\
\left\lfloor q_{u_{y}}\right\rfloor=\left\lfloor u_{y_{1}} u_{y_{2}} u_{y_{3}} u_{y_{4}} u_{y_{5}}\right\rfloor \\
\left\lfloor\delta q_{u_{y}}\right\rfloor=\left\lfloor\delta u_{y_{1}} \delta u_{y_{2}} \delta u_{y_{3}} \delta u_{y_{4}} \delta u_{y_{5}}\right\rfloor \\
\left\lfloor q_{w_{y}}\right\rfloor=\left\lfloor w_{y_{1}} w_{y_{2}} w_{y_{3}} w_{y_{4}} w_{y_{5}}\right\rfloor \\
\left\lfloor\delta q_{w_{y}}\right\rfloor=\left\lfloor\delta w_{y_{1}} \delta w_{y_{2}} \delta w_{y_{3}} \delta w_{y_{4}} \delta w_{y_{5}}\right\rfloor
\end{gathered}
$$

The stiffness matrix is formed by 25 sub matrices is obtained. The sub matrices for the elastic

part

are,

$\left[K_{u u}\right],\left[K_{u \gamma}\right],\left[K_{u w}\right],\left[K_{u u_{y}}\right],\left[K_{u w_{y}}\right]\left[K_{\gamma \gamma}\right],\left[K_{\gamma w}\right],\left[K_{\gamma u_{y}}\right],\left[K_{\gamma w_{y}}\right],\left[K_{w w}\right],\left[K_{w u_{y}}\right],\left[K_{w w_{y}}\right],\left[K_{u_{y} u_{y}}\right],\left[K_{u_{y} w_{y}}\right],[K$ with dimensions respectively of $5 \times 5,5 \times 5,5 \times 7,5 \times 5,5 \times 5,5 \times 5,5 \times 7,5 \times 5,5 \times 5,7 \times 7,7 \times 5,7 \times 5,5 \times 5$, $5 \times 5$ and $5 \times 5$.

The element stiffness matrix is given by,

$$
\left[K_{e}\right]=\left[\begin{array}{ccccc}
{\left[K_{u u}\right]} & {\left[K_{u u_{y}}\right]} & {\left[K_{u \gamma}\right]} & {\left[K_{u w}\right]} & {\left[K_{u w_{y}}\right]} \\
{\left[K_{u_{y} u}\right]} & {\left[K_{u_{y} u_{y}}\right]} & {\left[K_{u_{y} \gamma}\right]} & {\left[K_{u_{y} w}\right]} & {\left[K_{u_{y} w_{y}}\right]} \\
{\left[K_{\gamma u}\right]} & {\left[K_{\gamma u_{y}}\right]} & {\left[K_{\gamma \gamma}\right]} & {\left[K_{\gamma w}\right]} & {\left[K_{\gamma w_{y}}\right]} \\
{\left[K_{w u}\right]} & {\left[K_{w u_{y}}\right]} & {\left[K_{w \gamma}\right]} & {\left[K_{w w}\right]} & {\left[K_{w w_{y}}\right]} \\
{\left[K_{w_{y} u}\right]} & {\left[K_{w_{y} u_{y}}\right]} & {\left[K_{w_{y} \gamma}\right]} & {\left[K_{w_{y} w}\right]} & {\left[K_{w_{y} w_{y}}\right]}
\end{array}\right]
$$

\subsubsection{Stiffness Matrix for a Thin Curved Beam Element}

The sub matrices of Eq. (3.17) for the case a thin curved beam are given by, 


$$
\begin{aligned}
& \left.\left[K_{u u}\right]=b \int_{0}^{\alpha}\left(\frac{S_{11}}{R}\right)\left\{H_{L}^{\theta}\right\} \mid H_{L}^{\theta}\right] d \theta_{e} \\
& {\left[K_{u \gamma}\right]=b \int_{0}^{\alpha}\left(S_{12}-\frac{S_{14}}{R}\right)\left\{H_{L}^{\theta}\right\}\left\lfloor H_{L}\right\rfloor d \theta_{e}} \\
& {\left[K_{u w}\right]=b \int_{0}^{\alpha}\left[\left(\left(\frac{S_{11}}{R}-\frac{S_{13}}{R^{2}}\right)\left\{H_{L}^{\theta}\right\}\lfloor H\rfloor\right)-\left(\left(\frac{S_{13}}{R^{2}}\right)\left\{H_{L}^{\theta}\right\}\left\lfloor H^{\theta \theta}\right\rfloor\right)\right] d \theta_{e}} \\
& {\left[K_{u u^{y}}\right]=2 * b \int_{0}^{\alpha}\left(\frac{S_{14}}{R}\right)\left\{H_{L}^{\theta}\right\}\left\lfloor H_{L}\right\rfloor d \theta_{e}} \\
& \left.\left[K_{u w^{y}}\right]=-2 * b \int_{0}^{\alpha}\left(\frac{S_{14}}{R}\right)\left\{H_{L}^{\theta}\right\} \mid H_{L}^{\theta}\right\rfloor d \theta_{e} \\
& {\left[K_{\gamma \gamma}\right]=b \int_{0}^{\alpha}\left(R * S_{22}-S_{24}-S_{42}+\frac{S_{44}}{R}\right)\left\{H_{L}\right\}\left\lfloor H_{L}\right\rfloor d \theta_{e}} \\
& {\left[K_{\gamma w}\right]=b \int_{0}^{\alpha}\left[\left(\left(S_{21}-\frac{S_{23}}{R}-\frac{S_{41}}{R}+\frac{S_{43}}{R^{2}}\right)\left\{H_{L}\right\}\lfloor H\rfloor\right)-\left(\left(\frac{S_{23}}{R}-\frac{S_{43}}{R^{2}}\right)\left\{H_{L}\right\}\left\lfloor H^{\theta \theta}\right\rfloor\right)\right] d \theta_{e}} \\
& {\left[K_{\gamma u^{y}}\right]=2 * b \int_{0}^{\alpha}\left(S_{24}-\frac{S_{44}}{R}\right)\left\{H_{L}\right\}\left\lfloor H_{L}\right\rfloor d \theta_{e}} \\
& {\left[K_{\gamma w^{y}}\right]=-2 * b \int_{0}^{\alpha}\left(S_{24}-\frac{S_{44}}{R}\right)\left\{H_{L}\right\}\left[H^{\theta}{ }_{L}\right] d \theta_{e}}
\end{aligned}
$$




$$
\begin{aligned}
& {\left[K_{w w}\right]=b \int_{0}^{\alpha}\left[\left(\left(\frac{S_{11}}{R}-\frac{S_{13}}{R^{2}}-\frac{S_{31}}{R^{2}}+\frac{S_{33}}{R^{3}}\right)\{H\}\lfloor H\rfloor\right)\right.} \\
& -\left(\left(\frac{S_{13}}{R^{2}}-\frac{S_{33}}{R^{3}}\right)\{H\}\left\lfloor H^{\theta \theta}\right\rfloor\right)-\left(\left(\frac{S_{31}}{R^{2}}-\frac{S_{33}}{R^{3}}\right)\left\{H^{\theta \theta}\right\}\lfloor H\rfloor\right) \\
& \left.+\left(\frac{S_{33}}{R^{3}}\left\{H^{\theta \theta}\right\}\left[H^{\theta \theta}\right\rfloor\right)\right] d \theta_{e} \\
& {\left[K_{w u^{y}}\right]=2 * b \int_{0}^{\alpha}\left[\left(\left(\frac{S_{14}}{R}-\frac{S_{34}}{R^{2}}\right)\{H\}\left\lfloor H_{L}\right\rfloor\right)-\left(\frac{S_{34}}{R^{2}}\left\{H^{\theta \theta}\right\}\left\lfloor H_{L}\right\rfloor\right)\right] d \theta_{e}} \\
& \left.\left[K_{w w^{y}}\right]=-2 * b \int_{0}^{\alpha}\left[\left(\left(\frac{S_{14}}{R}-\frac{S_{34}}{R^{2}}\right)\{H\} \mid H_{L}^{\theta}\right\rfloor\right)-\left(\frac{S_{34}}{R^{2}}\left\{H^{\theta \theta}\right\}\left\lfloor H^{\theta}\right\rfloor\right)\right] d \theta_{e} \\
& {\left[K_{u^{y} u^{y}}\right]=4 * b \int_{0}^{\alpha} \frac{S_{44}}{R}\left\{H_{L}\right\}\left\lfloor H_{L}\right\rfloor d \theta_{e}} \\
& \left.\left[K_{u^{y} w^{y}}\right]=-4 * b \int_{0}^{\alpha} \frac{S_{44}}{R}\left\{H_{L}\right\} \mid H_{L}^{\theta}\right] d \theta_{e} \\
& {\left[K_{w^{y} w^{y}}\right]=4 * b \int_{0}^{\alpha} \frac{S_{44}}{R}\left\{H_{L}^{\theta}\right\}\left\lfloor H_{L}^{\theta}\right\rfloor d \theta_{e}}
\end{aligned}
$$




\subsubsection{Stiffness Matrix for Thick Curved Beam}

The sub matrices of Eq. (3.17) for the case a thick curved beam are given by,

$$
\begin{aligned}
& {\left[K_{u u}\right]=b \int_{0}^{\alpha_{e}}\left(\frac{S_{11}}{R}\right)\left\{H_{L}^{\theta}\right\}\left[H_{L}^{\theta}\right] d \theta_{e}} \\
& {\left[K_{u u^{y}}\right]=b \int_{0}^{\alpha_{e}}\left(\frac{2 S_{14}}{R}-\frac{S_{16}}{R^{2}}\right)\left\{H_{L}^{\theta}\right\}\left\lfloor H_{L}\right\rfloor d \theta_{e}} \\
& {\left[K_{u \gamma}\right]=b \int_{0}^{\alpha_{e}}\left[\left(S_{12}-\frac{S_{14}}{R}+\frac{S_{16}}{R^{2}}\right)\left\{H_{L}^{\theta}\right\}\left\lfloor H_{L}\right\rfloor\right] d \theta_{e}} \\
& {\left[K_{u w}\right]=b \int_{0}^{\alpha_{e}}\left[\left(\left(\frac{S_{11}}{R}-\frac{S_{13}}{R^{2}}+\frac{S_{15}}{R^{3}}\right)\left\{H_{L}^{\theta}\right\}\lfloor H\rfloor\right)+\left(\left(-\frac{S_{13}}{R^{2}}+\frac{S_{15}}{R^{3}}\right)\left\{H_{L}^{\theta}\right\}\left\lfloor H^{\theta \theta}\right\rfloor\right)\right] d \theta_{e}} \\
& {\left[K_{u w^{y}}\right]=b \int_{0}^{\alpha_{e}}\left(\frac{S_{16}}{R^{2}}-\frac{2 S_{14}}{R}\right)\left\{H_{L}^{\theta}\right\}\left[H_{L}^{\theta}\right\rfloor d \theta_{e}} \\
& {\left[K_{u^{y} u^{y}}\right]=b \int_{0}^{\alpha_{e}}\left(\frac{4 S_{44}}{R}-\frac{2 S_{46}}{R^{2}}-\frac{2 S_{64}}{R^{2}}+\frac{S_{66}}{R^{2}}\right)\left\{H_{L}\right\}\left\lfloor H_{L}\right\rfloor d \theta_{e}} \\
& {\left[K_{u^{y} \gamma}\right]=b \int_{0}^{\alpha_{e}}\left(2 S_{42}-\frac{2 S_{44}}{R}+\frac{2 S_{46}}{R^{2}}+\frac{S_{64}}{R^{2}}-\frac{S_{62}}{R}-\frac{S_{66}}{R^{3}}\right)\left\{H_{L}\right\}\left\lfloor H_{L}\right\rfloor d \theta_{e}} \\
& {\left[K_{u^{y_{w}}}\right]=b \int_{0}^{\alpha_{e}}\left[\left(\left(\frac{2 S_{41}}{R}-\frac{2 S_{43}}{R^{2}}+\frac{2 S_{45}}{R^{3}}-\frac{S_{61}}{R^{2}}+\frac{S_{63}}{R^{3}}-\frac{S_{65}}{R^{4}}\right)\left\{H_{L}\right\}\lfloor H\rfloor\right)\right.} \\
& \left.+\left(\left(\frac{2 S_{45}}{R^{3}}-\frac{2 S_{43}}{R^{2}}-\frac{S_{65}}{R^{4}}+\frac{S_{63}}{R^{3}}\right)\left\{H_{L}\right\}\left\lfloor H^{\theta \theta}\right\rfloor\right)\right] d \theta_{e}
\end{aligned}
$$




$$
\begin{aligned}
& \left.\left[K_{u^{y} w^{y}}\right]=b \int_{0}^{\alpha_{e}}\left(\frac{2 S_{46}}{R^{2}}-\frac{4 S_{44}}{R}-\frac{S_{66}}{R^{3}}+\frac{2 S_{64}}{R^{2}}\right)\left\{H_{L}\right\} \mid H_{L}^{\theta}\right\rfloor d \theta_{e} \\
& {\left[K_{\gamma \gamma}\right]=b \int_{0}^{\alpha_{e}}\left(R S_{22}-S_{24}+\frac{S_{26}}{R}-S_{42}+\frac{S_{44}}{R}-\frac{S_{46}}{R^{2}}+\frac{S_{62}}{R}-\frac{S_{64}}{R^{2}}\right.} \\
& \left.+\frac{S_{66}}{R^{3}}\right)\left\{H_{L}\right\}\left\lfloor H_{L}\right\rfloor d \theta_{e} \\
& {\left[K_{\gamma w}\right]=b \int_{0}^{\alpha_{e}}\left[\left(\left(S_{21}-\frac{S_{23}}{R}+\frac{S_{25}}{R^{2}}-\frac{S_{41}}{R}+\frac{S_{43}}{R^{2}}-\frac{S_{45}}{R^{3}}+\frac{S_{61}}{R^{2}}-\frac{S_{63}}{R^{3}}+\frac{S_{65}}{R^{4}}\right)\left\{H_{L}\right\}\lfloor H\rfloor\right)\right.} \\
& \left.+\left(\left(\frac{S_{25}}{R^{2}}-\frac{S_{23}}{R}-\frac{S_{45}}{R^{3}}+\frac{S_{43}}{R^{2}}+\frac{S_{65}}{R^{4}}-\frac{S_{63}}{R^{3}}\right)\left\{H_{L}\right\}\left[H^{\theta \theta}\right\rfloor\right)\right] d \theta_{e} \\
& \left.\left[K_{\gamma w^{y}}\right]=b \int_{0}^{\alpha_{e}}\left(\frac{S_{26}}{R}-2 S_{24}+\frac{2 S_{44}}{R}-\frac{S_{46}}{R^{2}}-\frac{2 S_{46}}{R^{2}}+\frac{S_{66}}{R^{3}}\right)\left\{H_{L}\right\} \mid H_{L}{ }^{\theta}\right] d \theta_{e} \\
& {\left[K_{w w}\right]=b \int_{0}^{\alpha_{e}}\left[\left(\left(-\frac{S_{13}}{R^{2}}+\frac{S_{15}}{R^{3}}+\frac{S_{11}}{R}-\frac{S_{31}}{R^{2}}+\frac{S_{33}}{R^{3}}-\frac{S_{35}}{R^{4}}+\frac{S_{51}}{R^{3}}-\frac{S_{53}}{R^{4}}+\frac{S_{55}}{R^{5}}\right)\{H\}\lfloor H\rfloor\right)\right.} \\
& +\left(\left(\frac{S_{15}}{R^{3}}-\frac{S_{13}}{R^{2}}-\frac{S_{35}}{R^{4}}+\frac{S_{33}}{R^{3}}+\frac{S_{55}}{R^{5}}-\frac{S_{53}}{R^{4}}\right)\{H\}\left\lfloor H^{\theta \theta}\right\rfloor\right) \\
& +\left(\left(\frac{S_{51}}{R^{3}}-\frac{S_{53}}{R^{4}}+\frac{S_{55}}{R^{5}}-\frac{S_{31}}{R^{2}}-\frac{S_{33}}{R^{3}}+\frac{S_{35}}{R^{4}}\right)\left\{H^{\theta \theta}\right\}\lfloor H\rfloor\right) \\
& \left.+\left(\left(\frac{S_{55}}{R^{5}}-\frac{S_{53}}{R^{4}}-\frac{S_{35}}{R^{4}}+\frac{S_{33}}{R^{3}}\right)\left\{H^{\theta \theta}\right\}\left[H^{\theta \theta}\right\rfloor\right)\right] d \theta_{e}
\end{aligned}
$$




$$
\begin{aligned}
& {\left[K_{w w^{y}}\right]=b \int_{0}^{\alpha_{e}} } {\left[\left(\left(\frac{S_{16}}{R^{2}}-\frac{2 S_{14}}{R}-\frac{S_{36}}{R^{3}}+\frac{2 S_{34}}{R^{2}}+\frac{S_{56}}{R^{4}}-\frac{2 S_{54}}{R^{3}}\right)\{H\}\left\lfloor H_{L}^{\theta}\right\rfloor\right)\right.} \\
&+\left.\left(\left(\frac{S_{56}}{R^{4}}-\frac{2 S_{54}}{R^{3}}-\frac{S_{36}}{R^{3}}+\frac{2 S_{34}}{R^{2}}\right)\left\{H^{\theta \theta}\right\}\left\lfloor H_{L}^{\theta}\right\rfloor\right)\right] d \theta_{e} \\
& {\left[K_{\left.w^{y} w^{y}\right]=b} \int_{0}^{\alpha_{e}}\left(\frac{S_{66}}{R^{3}}-\frac{2 S_{64}}{R^{2}}-\frac{2 S_{46}}{R^{2}}+\frac{4 S_{44}}{R}\right)\left\{H_{L}^{\theta}\right\}\left\lfloor H_{L}^{\theta}\right\rfloor d \theta_{e}\right.}
\end{aligned}
$$




\section{NUMERICAL IMPLEMENTATION}

\subsection{Introduction}

The finite element formulation of a composite curved beam has been presented in the previous chapter. The Gauss quadrature technique for numerical integration is discussed in this chapter. The beam is divided into several elements along the length of the beam and stiffness matrices are calculated using the above discussed technique. A computer program in MATLAB is written to solve the finite element equations.

\subsection{Numerical Integration in Space Domain}

The element stiffness matrix computation can be carried out by numerical integration. The commonly used scheme is the Gauss quadrature technique. This scheme needs $n$ unequallyspaced sampling points to integrate a polynomial of order $(2 n-1)$ exactly. The highest order of polynomial representing the shape functions is seven. Considering a uniform beam, the highest order polynomial is 14 . Thus, a seven-point Gauss-quadrature scheme is adopted in the present research. The sampling points and their respective weights for a seven-point integration scheme are shown in Table 4.1. The Gauss integration scheme is given below.

$$
\int_{-1}^{1} f(\xi) d \xi=\sum_{i=1}^{n} w_{i} f\left(a_{i}\right)
$$


where $n$ is the number of sampling points, $a_{i}$ is the $\xi$ co-ordinate of a sampling point and $w_{i}$ is the corresponding weight.

Table 4:1: Sampling points and weights for seven-point Gauss Quadrature

\begin{tabular}{|c|c|}
\hline Sampling Points & Weights \\
\hline \pm 0.9491079123 & 0.1294849661 \\
\hline \pm 0.7415311855 & 0.2797053914 \\
\hline \pm 0.4058451513 & 0.3813005051 \\
\hline 0.0000000000 & 0.4179591836 \\
\hline
\end{tabular}

The stiffness matrix is numerically integrated and the limits are changed from $\left(0, I_{e}\right)$ to $(-1,1)$ by non-dimensionalizing the independent variable.

\subsection{Boundary conditions}

The boundary conditions corresponding to the several cases considered in this research are outlined below:

Hinged support: $u=w=w_{y}=0$

Fixed support: $u=w=w^{\prime}=w_{y}=0$

\subsection{Ply Stresses for Beams}

At a given cross section of the composite beam the procedure for determining the offaxis and on-axis ply stresses is given here. 
The strain components are obtained from the kinematics equations of the problem which are reproduced here.

$$
\begin{gathered}
\varepsilon_{\theta}=\varepsilon_{\theta}^{o}+z \chi_{\theta} \\
\varepsilon_{y}=\varepsilon_{y}^{o}+z \chi_{y} \\
\gamma_{\theta y}=\gamma_{\theta y}^{o}+z 2 \chi_{\theta y}
\end{gathered}
$$

The following steps outline the way to find the ply stresses at $\theta=\phi$ along the arc of the beam where $\theta$ is the distance from the left end of the beam to the point of interest. First, locate the element corresponding to $\theta=\phi$ and then find the following values for that element.

$$
\begin{gathered}
u_{\theta}(\phi)=\left[\begin{array}{llll}
H_{L 1}^{\prime}(\phi) & \ldots & H_{L 5}^{\prime}(\phi)
\end{array}\right]\left[\begin{array}{lll}
\bar{u}_{1} & \ldots & \bar{u}_{5}
\end{array}\right]^{T} \\
\gamma(\phi)=\left[\begin{array}{llll}
H_{L 1}(\phi) & \ldots & H_{L 5}(\phi)
\end{array}\right]\left[\begin{array}{lll}
\bar{\gamma}_{1} & \ldots & \bar{\gamma}_{5}
\end{array}\right]^{T} \\
w_{\theta}(\phi)=\left[\begin{array}{llll}
H_{1}^{\prime}(\phi) & \ldots & H_{5}^{\prime}(\phi)
\end{array}\right]\left[\begin{array}{llll}
\bar{w}_{1} & \ldots & \bar{w}_{5}^{\prime}
\end{array}\right]^{T} \\
w_{\theta \theta}(\phi)=\left[\begin{array}{lllll}
H_{1}^{\prime \prime}(\phi) & \ldots & H_{5}^{\prime \prime}(\phi)
\end{array}\right]\left[\begin{array}{llll}
\bar{w}_{1} & \ldots & \bar{w}^{\prime}
\end{array}\right]^{T} \\
u_{y}(\phi)=\left[\begin{array}{lllll}
H_{L 1}(\phi) & \ldots & H_{L 5}(\phi)
\end{array}\right]\left[\begin{array}{llll}
\bar{u}_{y_{1}} & \ldots & \bar{u}_{y_{5}}
\end{array}\right]^{T} \\
w_{\theta y}(\phi)=\left[\begin{array}{lllll}
H_{L 1}^{\prime}(\phi) & \ldots & H_{L 5}^{\prime}(\phi)
\end{array}\right]\left[\begin{array}{llll}
\bar{w}_{y_{1}} & \ldots & \bar{w}_{y_{5}}
\end{array}\right]^{T}
\end{gathered}
$$


Then the strains and curvatures are:

$$
\begin{gathered}
\varepsilon_{\theta}^{o}(\phi)=\frac{1}{R} u^{\theta}(\phi)+\frac{w(\phi)}{R} \\
\gamma_{\theta y}^{o}(\phi)=\gamma_{o}(\phi) \\
\chi_{\theta}(\phi)=-\frac{1}{R^{2}}\left(w(\phi)+w^{\theta \theta}(\phi)\right) \\
\chi_{\theta y}(\phi)=\frac{1}{R}\left(u^{y}(\phi)-\frac{\gamma_{o}(\phi)}{2}-w^{\theta y}(\phi)\right)
\end{gathered}
$$

Then we can find $\varepsilon_{y}^{o}(\phi)$ and $\chi_{y}(\phi)$ as,

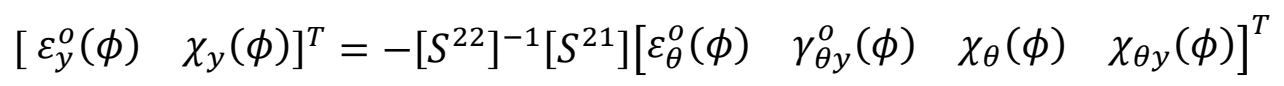

The ply strains at the top of the $k^{\text {th }}$ ply are represented as $\left[\begin{array}{ccc}\varepsilon_{\theta} & \varepsilon_{y} & \gamma_{\theta y}\end{array}\right]_{k t}$ and these strains are found using Eq. (4.4). Then the stresses at the top of the $k^{\text {th }}$ ply are,

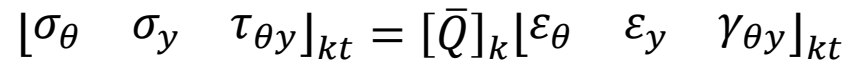

$$
\begin{aligned}
& \left\lfloor\begin{array}{lll}
\sigma_{1} & \sigma_{2} & \sigma_{6}
\end{array}\right\rfloor_{k t}=\left[T_{\sigma}\right]\left\lfloor\begin{array}{lll}
\sigma_{\theta} & \sigma_{y} & \tau_{\theta y}
\end{array}\right\rfloor_{k t}
\end{aligned}
$$

Similar to Eqs. (4.5) and (4.6), the ply strains and stresses are calculated for the bottom of the $k^{\text {th }}$ ply,

$$
\begin{aligned}
& {\left[\begin{array}{lll}
\sigma_{\theta} & \sigma_{y} & \tau_{\theta y}
\end{array}\right\rfloor_{k b}=[\bar{Q}]_{k}\left\lfloor\begin{array}{lll}
\varepsilon_{\theta} & \varepsilon_{y} & \gamma_{\theta y}
\end{array}\right\rfloor_{k b}} \\
& \left\lfloor\begin{array}{lll}
\sigma_{1} & \sigma_{2} & \sigma_{6}
\end{array}\right\rfloor_{k b}=\left[T_{\sigma}\right]\left\lfloor\begin{array}{lll}
\sigma_{\theta} & \sigma_{y} & \tau_{\theta y}
\end{array}\right\rfloor_{k b}
\end{aligned}
$$




\section{RESULTS AND DISCUSSION}

\subsection{Introduction}

A code is written in MATLAB to solve the finite element equations that are derived in the previous chapter. This code generates the necessary results in the form of displacements, forces, and stresses. Several examples, including isotropic and composite cases, are run to verify the formulation and compared with existing results from previous authors. Then some new results are generated.

\subsection{Verification of Curved Beam Results}

\subsubsection{Isotropic Beam Subjected to Point Load}

An isotropic curved beam in the form of a quarter circle is subjected to a point load in the negative radial direction at the top end and is fixed at the bottom end. The present results in the form of radial and tangential displacements are compared with the analytical results from Timoshenko and Goodier (1970). The geometric properties of the curved beam are,

Mid-plane radius $(R)=0.1 \mathrm{~m}$

Beam curved length $(L)=R \Theta=0.1 * \frac{\pi}{2} \mathrm{~m}$

Beam thickness $(t)=0.02 \mathrm{~m}$

Beam width $(b)=0.04 \mathrm{~m}$

$\operatorname{Load}(P)=1000 \mathrm{~N}$

The material properties correspond to Aluminium 6061-T6: 
Young's modulus $=E=68.9 \mathrm{GPa}$

Poisson's ratio $=v=0.33$

Shear modulus $=G=26 \mathrm{GPa}$

A convergence study is performed by varying the no of elements from 1 to 4 and comparing with the exact solution.

The exact solutions for the radial displacement $(w)$ and tangential displacement $(u)$ are taken from (Timoshenko \& Goodier, 1970) and rewritten here in terms of the radial coordinate $r$ and tangential coordinate $\theta$ :

$$
\begin{gathered}
w=-\frac{2 D}{E} \theta \cos \theta+\frac{\sin \theta}{E}\left[D(1-v) \log R+A(1-3 v) r^{2}+\frac{B(1+v)}{r^{2}}\right] \\
+K \sin \theta+L \cos \theta \\
u=\frac{2 D}{E} \theta \cos \theta-\frac{\cos \theta}{E}\left[-D(1-v) \log R+A(5+v) r^{2}+\frac{B(1+v)}{r^{2}}\right] \\
+\frac{D(1+v)}{E} \cos \theta+K \cos \theta-L \sin \theta+H r
\end{gathered}
$$

where the constants $A, B, D, K, L$, and $N$ are (with $R_{i}$ and $R_{o}$ as the internal and external radii, respectively of the curved beam),

$$
\begin{gathered}
A=\frac{P}{2 N} \\
B=-\frac{P R_{i}{ }^{2} R_{o}{ }^{2}}{2 N}
\end{gathered}
$$




$$
\begin{gathered}
D=-\frac{P}{N}\left(R_{o}{ }^{2}+{R_{i}}^{2}\right) \\
N={R_{i}}^{2}-{R_{o}}^{2}+\left({R_{i}}^{2}+{R_{o}}^{2}\right) \log \frac{R_{o}}{R_{i}} \\
L=\frac{D \pi}{E} \\
K=-\frac{1}{E}\left(A(1-3 v) R^{2}+\frac{B(1+v)}{R^{2}}+D(1+v) \log R\right)
\end{gathered}
$$

\subsubsection{Radial Displacement}

The radial displacement values obtained with 1 to 4 elements under the thin curved beam approximation (first order) are presented in Table.5.1 and under the thick curved beam approximation (second order) are presented in Table 5.2. The exact solution from Timoshenko and Goodier is also presented in these tables. There is not much of variation in the results in the convergence study from 2 to 4 elements but we have decided to keep 4 elements since this will give more data points for obtaining the nodal forces and moments. The comparison with the exact solution is very good. Another observation is that difference between the results of the first order and second order formulations is very small for this example.

Table 5:1 Radial displacement for first order formulation

\begin{tabular}{|c|c|c|c|c|c|}
\hline \multicolumn{6}{|c|}{$w(\mathrm{~m})$} \\
\hline Angle & 1 Element & 2 Element & 3 Element & 4 Element & Exact \\
\hline 0.0 & $-4.2705 \mathrm{E}-04$ & $-4.2745 \mathrm{E}-04$ & $-4.2745 \mathrm{E}-04$ & $-4.2745 \mathrm{E}-04$ & $-4.3001 \mathrm{E}-04$ \\
\hline 22.5 & $-2.9615 \mathrm{E}-04$ & $-2.9618 \mathrm{E}-04$ & $-2.9618 \mathrm{E}-04$ & $-2.9618 \mathrm{E}-04$ & $-2.9795 \mathrm{E}-04$ \\
\hline 45.0 & $-1.5062 \mathrm{E}-04$ & $-1.5112 \mathrm{E}-04$ & $-1.5112 \mathrm{E}-04$ & $-1.5112 \mathrm{E}-04$ & $-1.5203 \mathrm{E}-04$ \\
\hline 67.5 & $-4.0695 \mathrm{E}-05$ & $-4.0892 \mathrm{E}-05$ & $-4.0893 \mathrm{E}-05$ & $-4.0894 \mathrm{E}-05$ & $-4.1139 \mathrm{E}-05$ \\
\hline 90.0 & $0.0000 \mathrm{E}+00$ & $0.0000 \mathrm{E}+00$ & $0.0000 \mathrm{E}+00$ & $0.0000 \mathrm{E}+00$ & $0.0000 \mathrm{E}+00$ \\
\hline
\end{tabular}


Table 5:2 Radial displacement for Second order formulation

\begin{tabular}{|c|c|c|c|c|c|}
\hline \multicolumn{6}{|c|}{$\boldsymbol{w}(\boldsymbol{m})$} \\
\hline Angle & 1 Element & 2 Element & 3 Element & 4 Element & Exact \\
\hline 0.0 & $-4.2726 \mathrm{E}-04$ & $-4.2745 \mathrm{E}-04$ & $-4.2745 \mathrm{E}-04$ & $-4.2745 \mathrm{E}-04$ & $-4.3001 \mathrm{E}-04$ \\
\hline 22.5 & $-2.9658 \mathrm{E}-04$ & $-2.9618 \mathrm{E}-04$ & $-2.9665 \mathrm{E}-04$ & $-2.9669 \mathrm{E}-04$ & $-2.9796 \mathrm{E}-04$ \\
\hline 45.0 & $-1.5162 \mathrm{E}-04$ & $-1.5113 \mathrm{E}-04$ & $-1.5118 \mathrm{E}-04$ & $-1.5126 \mathrm{E}-04$ & $-1.5203 \mathrm{E}-04$ \\
\hline 67.5 & $-4.1096 \mathrm{E}-05$ & $-4.0983 \mathrm{E}-05$ & $-4.0992 \mathrm{E}-05$ & $-4.0995 \mathrm{E}-05$ & $-4.1139 \mathrm{E}-05$ \\
\hline 90.0 & $0.0000 \mathrm{E}+00$ & $0.0000 \mathrm{E}+00$ & $0.0000 \mathrm{E}+00$ & $0.0000 \mathrm{E}+00$ & $0.0000 \mathrm{E}+00$ \\
\hline
\end{tabular}

\subsubsection{Tangential Displacement}

The tangential displacement values obtained with 1 to 4 elements under the thin curved beam approximation (first order) are presented in Table.5.3 and under the thick curved beam approximation (second order) are presented in Table 5.4. The exact solution from Timoshenko and Goodier is also presented in these tables. We can make similar observations here as that for the radial displacement.

Table 5:3 Tangential displacement for first order formulation

\begin{tabular}{|c|c|c|c|c|c|}
\hline \multicolumn{6}{|c|}{$u(\mathbf{m})$} \\
\hline Angle & 1 Element & 2 Element & 3 Element & 4 Element & Exact \\
\hline 0.0 & $-2.7231 \mathrm{E}-04$ & $-2.7212 \mathrm{E}-04$ & $-2.7212 \mathrm{E}-04$ & $-2.7213 \mathrm{E}-04$ & $-2.75 \mathrm{E}-04$ \\
\hline 22.5 & $-1.2882 \mathrm{E}-04$ & $-1.2824 \mathrm{E}-04$ & $-1.2828 \mathrm{E}-04$ & $-1.2873 \mathrm{E}-04$ & $-1.30 \mathrm{E}-04$ \\
\hline 45.0 & $-4.1165 \mathrm{E}-05$ & $-4.1292 \mathrm{E}-05$ & $-4.1291 \mathrm{E}-05$ & $-4.1293 \mathrm{E}-05$ & $-4.22 \mathrm{E}-05$ \\
\hline 67.5 & $-5.0652 \mathrm{E}-06$ & $-5.3636 \mathrm{E}-06$ & $-5.3980 \mathrm{E}-06$ & $-5.4086 \mathrm{E}-06$ & $-5.41 \mathrm{E}-06$ \\
\hline 90.0 & $0.0000 \mathrm{E}+00$ & $0.0000 \mathrm{E}+00$ & $0.0000 \mathrm{E}+00$ & $0.0000 \mathrm{E}+00$ & $0.00 \mathrm{E}+00$ \\
\hline
\end{tabular}


Table 5:4 Tangential displacement for second order formulation

\begin{tabular}{|c|c|c|c|c|c|}
\hline \multicolumn{6}{|c|}{$\boldsymbol{u}(\boldsymbol{m})$} \\
\hline Angle & 1 Element & 2 Element & 3 Element & 4 Element & Exact \\
\hline 0.0 & $-2.7231 \mathrm{E}-04$ & $-2.7212 \mathrm{E}-04$ & $-2.7212 \mathrm{E}-04$ & $-2.7213 \mathrm{E}-04$ & $-2.7464 \mathrm{E}-04$ \\
\hline 22.5 & $-1.2971 \mathrm{E}-04$ & $-1.2878 \mathrm{E}-04$ & $-1.2828 \mathrm{E}-04$ & $-1.2873 \mathrm{E}-04$ & $-1.3031 \mathrm{E}-04$ \\
\hline 45.0 & $-4.1565 \mathrm{E}-05$ & $-4.16215 \mathrm{E}-05$ & $-4.1984 \mathrm{E}-05$ & $-4.1985 \mathrm{E}-05$ & $-4.2167 \mathrm{E}-05$ \\
\hline 67.5 & $-5.1642 \mathrm{E}-06$ & $-5.37851 \mathrm{E}-06$ & $-5.3980 \mathrm{E}-06$ & $-5.4097 \mathrm{E}-06$ & $-5.4102 \mathrm{E}-06$ \\
\hline 90.0 & $0.0000 \mathrm{E}+00$ & $0.0000 \mathrm{E}+00$ & $0.0000 \mathrm{E}+00$ & $0.0000 \mathrm{E}+00$ & $0.0000 \mathrm{E}+00$ \\
\hline
\end{tabular}

\subsubsection{Reaction Forces}

The reactions are calculated and checked against expected values from statics. The radial and moment reactions have agreed excellently with the exact values from statics while that for the tangential reaction have differed considerably. A number of simulations and checks have not pinpointed the reason for this anomaly since all other results such as displacements, shear forces, bending moments, and stresses agree excellently where exact results are available. For the record, the reactions obtained by the models and the corresponding exact values for two examples are given below.

Table 5:5 Reaction Forces

\begin{tabular}{|c|c|c|c|c|c|c|}
\hline & \multicolumn{2}{|c|}{ Shear Force $(\mathrm{N})$} & \multicolumn{2}{c|}{ Moment $(\mathrm{Nm})$} & \multicolumn{2}{c|}{ Axial Force $(\mathrm{N})$} \\
\hline Angle & Exact & FEM & Exact & FEM & Exact & FEM \\
\hline 0 & 0 & $-1.23 \mathrm{E}-09$ & 0 & $1.86 \mathrm{E}-10$ & -1000.000 & -1000 \\
\hline 22.5 & -382.683 & -382.683 & -7.23144 & $-7.23 \mathrm{E}+00$ & -923.880 & -1000 \\
\hline 45 & -707.107 & -707.107 & -27.8249 & -27.8249 & -707.107 & -1000 \\
\hline 67.5 & -923.88 & -923.88 & -58.6451 & -58.6451 & -382.683 & -1000 \\
\hline 90 & -1000 & 1000 & -95 & -95 & 0.000 & 1000 \\
\hline
\end{tabular}




\subsubsection{Isotropic Beam Subjected to Bending Moment}

(Nguyen, 2010) considers a small curved beam (Fig. 5.1) and models it in ABACUS; he presents results in the form of the variation of the tangential stress with respect to the radial position $r$. This model is duplicated in our code to verify the tangential stress distribution; the distribution is also compared with the exact solution from (Timoshenko \& Goodier, 1970).

The geometric properties of the curved beam ring are as follows,

Mid-plane radius $(R)=0.6444$ in.

Inner radius $\left(R_{i}\right)=0.6 \mathrm{in}$.

Outer radius $\left(R_{0}\right)=0.6888$.

Beam curved length $(L)=R \theta=0.6444 * \frac{\pi}{2}$ in

Beam thickness $(h)=0.0888$ in.

Beam width $(b)=1.0$ in.

Applied moment $(M)=100 \mathrm{lb}$-in

Aluminium Al-2014-T6 material properties:

Young's modulus $=E=10.6 \mathrm{Msi}$

Poisson's ratio $=v=0.35$

Shear modulus $=G=3.9 \mathrm{Msi}$

The formula used for calculating the tangential stress is.

$$
\sigma_{\theta}=-\frac{4 M}{N}\left(-\frac{R_{i}^{2} R_{o}^{2}}{R^{2}} \log \frac{R_{o}}{R_{i}}+R_{o}^{2} \log \frac{R}{R_{o}}+R_{i}^{2} \log \frac{R_{i}}{R}+R_{o}^{2}-R_{i}^{2}\right)
$$

where 


$$
N=\left(R_{o}^{2}-R_{i}^{2}\right)^{2}-4 R_{i}^{2} R_{o}^{2}\left(\log \frac{R_{o}}{R_{i}}\right)^{2}
$$

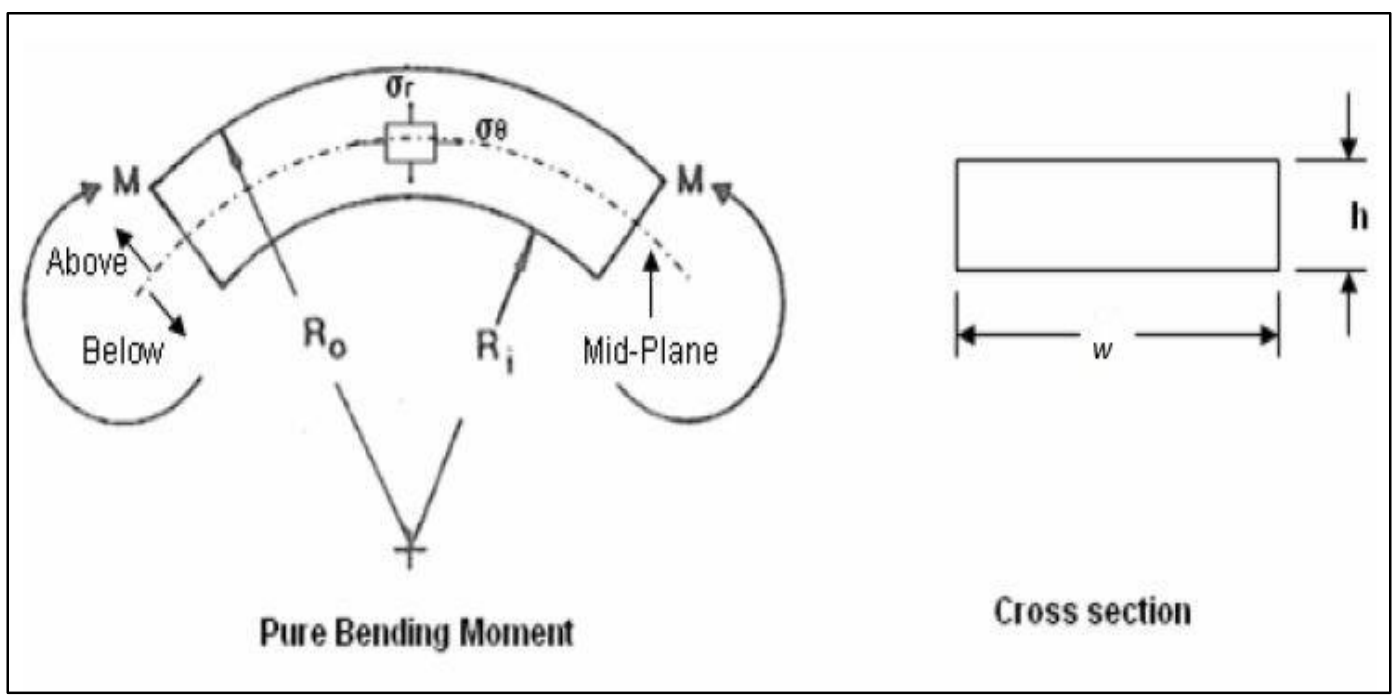

Fig 5-1: Curved beam subjected to bending moment and its cross section. (Nguyen, 2010)

The results obtained from the present model for the tangential stress are presented in Table 5.5. The results of Nguyen and the exact solution are also shown in this table. The comparison indicates that the present results are more accurate than that of Nguyen when compared with the exact solution. The comparison is also shown graphically in Fig. 5.2.

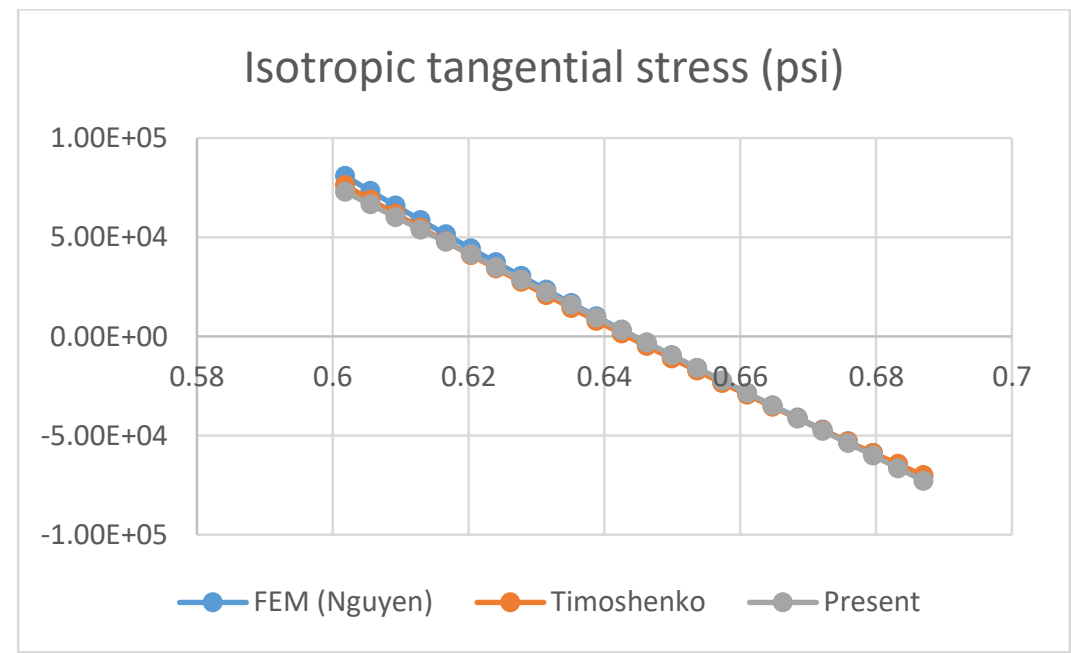

Fig 5-2: Tangential stress comparison 
Table 5:6: Tangential Stress Values for Isotropic Material

\begin{tabular}{|c|c|c|c|}
\hline \multicolumn{4}{|c|}{$\sigma_{\theta}(p s i)$} \\
\hline Radial Position (in.) & $\begin{array}{c}\text { FEM } \\
\text { (Nguyen) }\end{array}$ & Timoshenko & Present \\
\hline 0.60185 & $8.08 \mathrm{E}+04$ & $7.61 \mathrm{E}+04$ & $7.28 \mathrm{E}+04$ \\
\hline 0.60555 & $7.33 E+04$ & $6.89 \mathrm{E}+04$ & $6.65 E+04$ \\
\hline 0.60925 & $6.59 \mathrm{E}+04$ & $6.18 \mathrm{E}+04$ & $6.02 E+04$ \\
\hline 0.61295 & $5.86 \mathrm{E}+04$ & $5.47 \mathrm{E}+04$ & $5.38 \mathrm{E}+04$ \\
\hline 0.61665 & $5.14 \mathrm{E}+04$ & $4.78 \mathrm{E}+04$ & $4.76 \mathrm{E}+04$ \\
\hline 0.62035 & $4.43 E+04$ & $4.09 E+04$ & $4.13 E+04$ \\
\hline 0.62405 & $3.73 E+04$ & $3.42 E+04$ & $3.49 E+04$ \\
\hline 0.62775 & $3.04 \mathrm{E}+04$ & $2.74 \mathrm{E}+04$ & $2.86 \mathrm{E}+04$ \\
\hline 0.63145 & $2.35 \mathrm{E}+04$ & $2.08 \mathrm{E}+04$ & $2.22 \mathrm{E}+04$ \\
\hline 0.63515 & $1.67 \mathrm{E}+04$ & $1.43 E+04$ & $1.59 \mathrm{E}+04$ \\
\hline 0.63885 & $1.00 \mathrm{E}+04$ & $7.82 E+03$ & $9.52 \mathrm{E}+03$ \\
\hline 0.64255 & $3.39 \mathrm{E}+03$ & $1.42 \mathrm{E}+03$ & $3.17 \mathrm{E}+03$ \\
\hline 0.64625 & $-3.15 E+03$ & $-4.90 \mathrm{E}+03$ & $-3.18 \mathrm{E}+03$ \\
\hline 0.64995 & $-9.63 E+03$ & $-1.11 E+04$ & $-9.54 \mathrm{E}+03$ \\
\hline 0.65365 & $-1.60 \mathrm{E}+04$ & $-1.73 E+04$ & $-1.59 \mathrm{E}+04$ \\
\hline 0.65735 & $-2.24 \mathrm{E}+04$ & $-2.34 \mathrm{E}+04$ & $-2.22 \mathrm{E}+04$ \\
\hline 0.66105 & $-2.86 \mathrm{E}+04$ & $-2.95 \mathrm{E}+04$ & $-2.86 \mathrm{E}+04$ \\
\hline 0.66475 & $-3.48 \mathrm{E}+04$ & $-3.54 \mathrm{E}+04$ & $-3.50 \mathrm{E}+04$ \\
\hline 0.66845 & $-4.09 E+04$ & $-4.14 \mathrm{E}+04$ & $-4.13 E+04$ \\
\hline 0.67215 & $-4.70 E+04$ & $-4.72 E+04$ & $-4.77 E+04$ \\
\hline 0.67585 & $-5.30 E+04$ & $-5.30 E+04$ & $-5.38 \mathrm{E}+04$ \\
\hline 0.67955 & $-5.89 E+04$ & $-5.87 E+04$ & $-6.02 E+04$ \\
\hline 0.68325 & $-6.48 \mathrm{E}+04$ & $-6.44 \mathrm{E}+04$ & $-6.65 E+04$ \\
\hline 0.68695 & $-7.06 E+04$ & $-7.00 E+04$ & $-7.28 \mathrm{E}+04$ \\
\hline
\end{tabular}




\subsubsection{Composite Pinched Ring}

Consider a composite pinched ring is subjected to a compressive point load in the radial direction as shown in Fig. 5.3. Since the ring has a double symmetry, only the quadrant measured from $A$ to $B$ is modeled. and thereby the load is half of what is being applied.

The geometric properties of the ring are:

$$
\begin{aligned}
& \text { Mid-plane radius }(R)=0.1 \mathrm{~m} \\
& \text { Beam curved length }(L)=R \Theta=0.1 * \frac{\pi}{2} \mathrm{~m} \\
& \text { Beam thickness }(t)=0.02 \mathrm{~m} \\
& \text { Beam width }(b)=0.02 \mathrm{~m} \\
& \text { Load }(P)=1 \mathrm{kN} \\
& \text { Stacking sequence }=[90 / 45 /-45 / 45 /-45] \mathrm{s} \\
& \text { The ply properties of the graphite-epoxy composite are, } \\
& E_{1}=289 \mathrm{GPa} \\
& E_{2}=6.06 \mathrm{GPa} \\
& G_{12}=G_{13}=G_{23}=4.13 \mathrm{GPa} \\
& V_{12}=0.31
\end{aligned}
$$

The shear force at the end nodes of each element are calculated using the element equations and presented as normalized values (with respect to the applied load of $1 \mathrm{kN}$ ) in Table 5.6. For comparison purposes, the results from Kim (2005) and the exact solution are also presented; the exact solution is from statics and results in $V=P \operatorname{Cos} \theta$. The present results are in excellent agreement with the other two sets. 


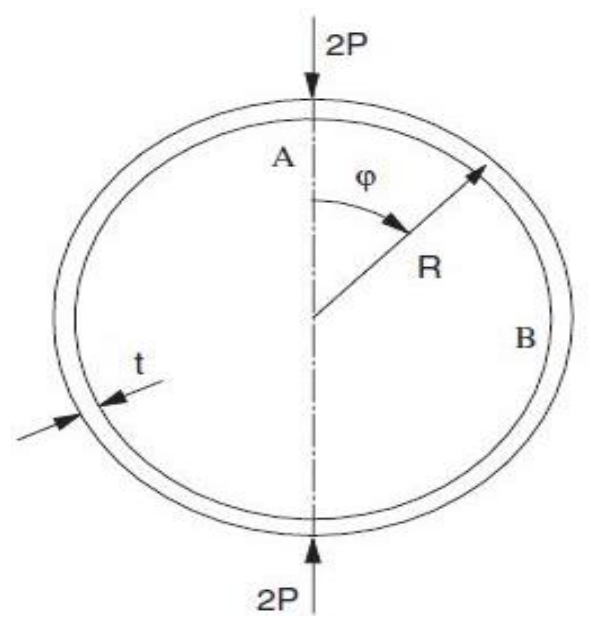

Fig 5-3: Composite pinched ring under compressive point load. (Kim, 2005)

In the present model, the curved beam is divided into 4 elements with 3 internal nodes per each element. The beam is subjected to a point load of $1 \mathrm{KN}$ at point $A$ and is fixed at point $B$ as shown in Fig. 5.4.

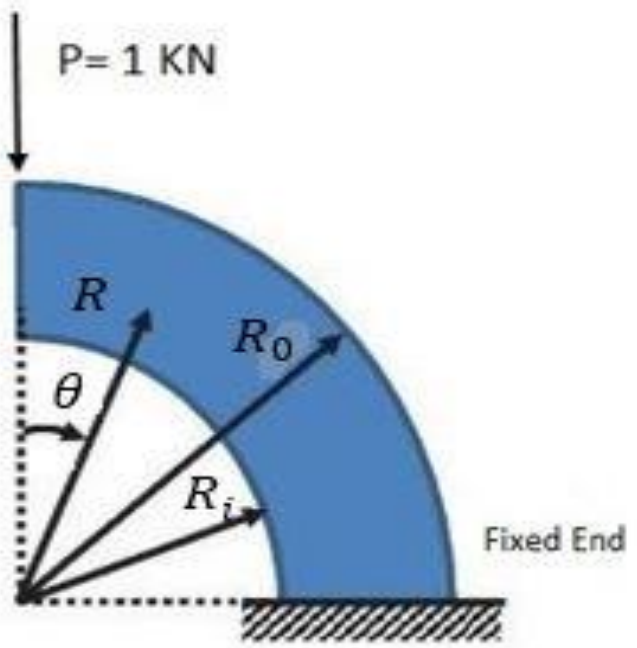

Fig 5-4: Curved beam subjected to loading conditions 
Table 5:7 Comparison of normalized shear force in pinched ring

\begin{tabular}{|c|c|c|c|c|}
\hline Angle & $\begin{array}{c}\text { Kim, } \\
2005\end{array}$ & $\begin{array}{c}\text { Present (1st } \\
\text { Order) }\end{array}$ & $\begin{array}{c}\text { Present } \\
\text { (2nd Order) }\end{array}$ & Exact \\
\hline 0 & -1.0000 & -1.0000 & -1.0000 & -1.0000 \\
\hline 22.5 & -0.9230 & -0.9239 & -0.9239 & -0.9240 \\
\hline 45 & -0.7087 & -0.7071 & -0.7073 & -0.7074 \\
\hline 67.5 & -0.3824 & -0.3827 & -0.3831 & -0.3832 \\
\hline 90 & 0.0000 & 0.0000 & 0.0000 & -0.0008 \\
\hline
\end{tabular}

\subsubsection{Stresses in a Curved Composite Beam Subjected to Bending Moment}

The curved beam of Fig. 5.1 is now made of a composite laminate. An angular length of $90^{\circ}$ is modelled with one end fixed and the other end subjected to a moment.

The geometric properties of the curved beam are:

Mid-plane radius $(R)=0.6444 \mathrm{in}$.

Beam curved length $(L)=R \Theta=0.6444 * \frac{\pi}{2}$ in

Beam thickness $(t)=0.0888$ in.

Beam width $(b)=1$ in.

Stacking Sequence $=\left[45 /-45 / 90_{2} / 0_{2}\right]_{\mathrm{s}}$

The ply properties of the graphite-epoxy composite are,

$$
\begin{aligned}
& E_{1}=21.75 \mathrm{Msi} \\
& E_{2}=1.595 \mathrm{Msi} \\
& G_{12}=G_{13}=0.8702 \mathrm{Msi} \\
& G_{23}=0.5366 \mathrm{Msi} \\
& \mathrm{v}_{12}=0.25
\end{aligned}
$$




$$
\operatorname{Load}(P)=1000 \mathrm{~N}
$$

The results obtained for the tangential stresses are provided in Table 5.7 and as a plot in Fig. 5.5. The orientation of the fibre and the radial positon both influence the tangential stress distribution.

Table 5:8: Tangential stress variation along radial position

\begin{tabular}{|c|c|c|}
\hline \multicolumn{3}{|c|}{$\sigma_{\theta}$ (psi) } \\
\hline $\begin{array}{c}\text { Layer } \\
\text { No }\end{array}$ & $\begin{array}{c}\text { Radial Position } \\
\text { (in.) }\end{array}$ & $\begin{array}{c}\text { Tangential } \\
\text { Stress }\end{array}$ \\
\hline 1 & 0.6037 & $6.74 \mathrm{E}+04$ \\
\hline 2 & 0.6111 & $6.83 \mathrm{E}+04$ \\
\hline 3 & 0.6185 & $1.37 \mathrm{E}+04$ \\
\hline 4 & 0.6259 & $9.82 \mathrm{E}+03$ \\
\hline 5 & 0.6333 & $8.75 \mathrm{E}+04$ \\
\hline 6 & 0.6407 & $2.91 \mathrm{E}+04$ \\
\hline 7 & 0.6481 & $-2.93 \mathrm{E}+04$ \\
\hline 8 & 0.6555 & $-8.77 \mathrm{E}+04$ \\
\hline 9 & 0.6629 & $-9.83 \mathrm{E}+03$ \\
\hline 10 & 0.6703 & $-1.38 \mathrm{E}+04$ \\
\hline 11 & 0.6777 & $-6.84 \mathrm{E}+04$ \\
\hline 12 & 0.6851 & $-6.74 \mathrm{E}+04$ \\
\hline
\end{tabular}




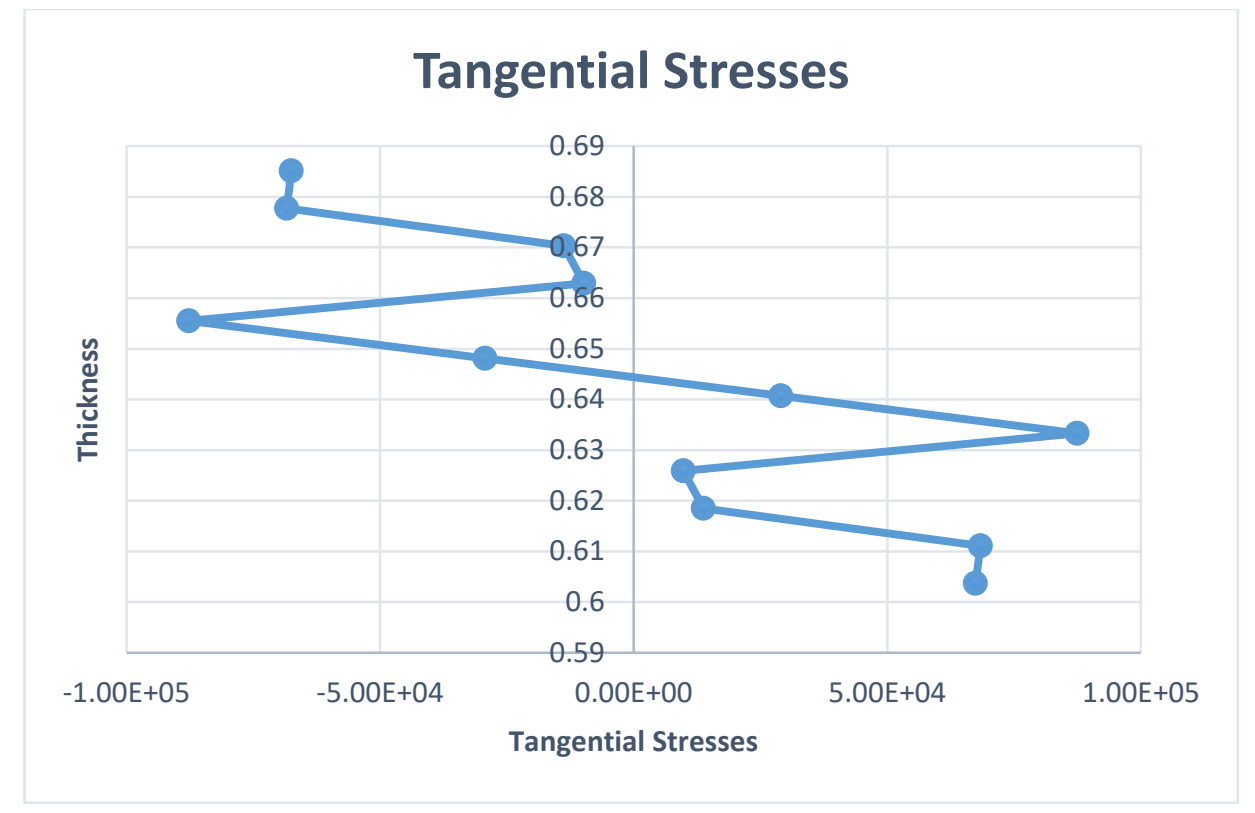

Fig 5.5: Tangential Stress plot along the varying Radial Position

\subsection{Present Results}

This section deals with new results generated on a pinned-pinned composite curved beam subjected to uniform loading conditions with various symmetrical and non-symmetrical layup configurations. The beam is of a rectangular cross section with the following properties:

The geometric properties of the ring are as follows,

Beam length $(L)=1 \mathrm{~m}$

Beam thickness $(t)=0.05 \mathrm{~m}$

Beam width $(b)=0.025 \mathrm{~m}$

The ply properties of the graphite-epoxy composite are,

$$
\begin{aligned}
& \mathrm{E}_{1}=138 \mathrm{GPa} \\
& \mathrm{E}_{2}=8.96 \mathrm{GPa} \\
& \mathrm{G}_{12}=\mathrm{G} 13=7.1 \mathrm{GPa}
\end{aligned}
$$




$$
\begin{aligned}
& \mathrm{G}_{23}=5.16 \mathrm{GPa} \\
& v_{12}=0.3
\end{aligned}
$$

A parametric study is carried out by varying the mean radius of the curved beam (while keeping the mean curved length a constant) and considering five different lay ups. The maximum deflection and bending moment are given in Tables 5.8 through 5.11 for the first-order and second-order formulations.

Table 5:9: Maximum deflection and moment of simply supported curved beam First Order

\begin{tabular}{|c|c|c|c|c|c|c|c|c|}
\hline \multicolumn{9}{|c|}{ First Order } \\
\hline \multicolumn{1}{|c|}{ Beam Properties } & \multicolumn{2}{|c|}{$\left[0_{4}\right]$} & \multicolumn{2}{c|}{$[0 / 90]_{\mathrm{s}}$} & \multicolumn{2}{c|}{$\left[0_{2} / 90_{2}\right]_{\mathrm{s}}$} \\
\hline$L(m)$ & $R(m)$ & $L / R$ & $w(m)$ & $M(N m)$ & $w(m)$ & $M(N m)$ & $w(m)$ & $M(N m)$ \\
\hline 1 & 5 & 0.2 & 0.000308 & 120.628193 & 0.000504 & 159.356790 & 0.000522131 & 159.46494 \\
\hline 1 & 1.67 & 0.6 & 0.000055 & 19.976136 & 0.000094 & 31.789563 & 0.000106286 & 31.928637 \\
\hline 1 & 1 & 1 & 0.000054 & 7.348292 & 0.000039 & 11.836982 & $4.00968 \mathrm{E}-05$ & 11.889163 \\
\hline 1 & 0.5 & 2 & 0.000005 & 1.581976 & 0.000010 & 2.449163 & $9.64917 \mathrm{E}-06$ & 2.5467982 \\
\hline
\end{tabular}

Table 5:10: Maximum deflection and moment of simply supported curved beam Second Order

\begin{tabular}{|c|c|c|c|c|c|c|c|c|}
\hline \multicolumn{9}{|c|}{ Second Order } \\
\hline \multicolumn{2}{|c|}{ Beam Properties } & \multicolumn{2}{|c|}{$\left[0_{4}\right]$} & \multicolumn{2}{c|}{$[0 / 90] \mathrm{s}$} & \multicolumn{2}{c|}{$\left[0_{2} / 90_{2}\right] \mathrm{s}$} \\
\hline$L(m)$ & $R(m)$ & $L / R$ & $w(m)$ & $M(N m)$ & $w(m)$ & $M(N m)$ & $w(m)$ & $M(N m)$ \\
\hline 1 & 5 & 0.2 & 0.000308 & 120.628193 & 0.000504 & 159.356790 & 0.000522131 & 159.46494 \\
\hline 1 & 1.67 & 0.6 & 0.000055 & 19.976136 & 0.000094 & 31.789563 & 0.000106286 & 31.928637 \\
\hline 1 & 1 & 1 & 0.000054 & 7.348292 & 0.000039 & 11.836982 & $4.00968 \mathrm{E}-05$ & 11.889163 \\
\hline 1 & 0.5 & 2 & 0.000005 & 1.581976 & 0.000010 & 2.449163 & $9.64917 \mathrm{E}-06$ & 2.5467982 \\
\hline
\end{tabular}


Table 5:11: Maximum deflection and moment of simply supported curved beam First Order

\begin{tabular}{|c|c|c|c|c|c|c|}
\hline \multicolumn{9}{|c|}{ First Order } \\
\hline \multicolumn{3}{|c|}{ Beam Properties } & \multicolumn{3}{c|}{$\left[45_{4}\right]$} & \multicolumn{2}{c|}{$[30 / 30 / 60 / 60]$} \\
\hline$L(m)$ & $R(m)$ & $L / R$ & $w(m)$ & $M(N m)$ & $w(m)$ & $M(N m)$ \\
\hline 1 & 5 & 0.2 & 0.00290 & 120.63974 & 0.00326 & 104.78946 \\
\hline 1 & 1.67 & 0.6 & 0.00051 & 20.25929 & 0.00044 & 4.20849 \\
\hline 1 & 1 & 1 & 0.00019 & 7.50089 & 0.00015 & 8.13805 \\
\hline 1 & 0.5 & 2 & 0.00005 & 1.76984 & 0.00003 & 6.02910 \\
\hline
\end{tabular}

Table 5:12: Maximum deflection and moment of simply supported curved beam Second Order

\begin{tabular}{|c|c|c|c|c|c|c|}
\hline \multicolumn{7}{|c|}{ Second Order } \\
\hline \multicolumn{2}{|c|}{ Beam Properties } & \multicolumn{2}{|c|}{$\left[45_{4}\right]$} & \multicolumn{2}{c|}{$[30 / 30 / 60 / 60]$} \\
\hline$L(m)$ & $R(m)$ & $L / R$ & $w(m)$ & $M(N m)$ & $w(m)$ & $M(N m)$ \\
\hline 1 & 5 & 0.2 & 0.00308 & 120.65116 & 0.00334 & 104.95596 \\
\hline 1 & 1.67 & 0.6 & 0.00052 & 20.27765 & 0.00045 & 4.23736 \\
\hline 1 & 1 & 1 & 0.00020 & 7.52226 & 0.00015 & 8.14671 \\
\hline 1 & 0.5 & 2 & 0.00005 & 1.79574 & 0.00003 & 6.03018 \\
\hline
\end{tabular}




\section{CONCLUSIONS \& RECOMMENDATIONS}

\subsection{Contributions}

1. A curved beam formulation is made based on the bending theory of cylindrical shells

2. A higher-order finite element model with five nodes and 27 degrees of freedom is presented for the analysis of a composite curved beam based on CLPT.

3. A higher-order shell theory formulation is adapted which can be applied to thick curved beams.

4. The process of static condensation is used to eliminate transverse strain and curvature for a composite curved beam.

5. A MATLAB code is written to solve the finite element equations and is used to compute the displacement, forces, moments and stresses in a composite curved beam.

\subsection{Conclusions}

1. For the curved beam examples considered, the results for displacements, shear forces, bending moments, and tangential stresses agreed excellently with that of previous authors and with the exact solution.

2. The results for the tangential reactions did not agree with the expected solution.

\subsection{Recommendations}

1. Formulate and solve the problem with other theories such as the first order shear deformation theory or higher order shear deformation theory to account for transverse shear effects. 
2. Consider curved beams that are not shaped in the form of a circular arc.

3. A dynamic analysis could be performed.

4. The numerical results could be experimentally validated. 


\section{REFERENCES}

Barbero, E. J., 2010. Introduction to Composite Materials Design. 2nd ed. s.I.:CRC Press.

Borri, M., Ghiringhelli, G. L. \& Merlini, T., 1992. Composite Beam Analysis-Linear Analysis of Naturally Curved \& Twisted Anisotropic Beams. London: EUROPEAN RESEARCH OFFICE OF THE U. S. ARMY.

Chandrasekaran, G., 2000. Dynamic Analysis of a Composite Moving Beam, Master's

Thesis, West Virginia University. Morgantown(WV): s.n.

Ecsedi, I. \& Dluhi, K., 2005. A linear model for the static and dynamic analysis of non-

homogeneous curved beams. Applied Mathematical Modelling, Issue 29, p. 1211-1231.

Erkmen, R. E. \& Bradford, M. A., 2009. Nonlinear Elastic Analysis of Composite Beams Curved In-plan. Engineering Structures, pp. 1613-1324.

Flugge, W., 1966. Stresses in Shells. New York: Splinger-Verlag Inc..

Hajianmaleki, M. \& Qatu, M. S., 2011. Advances in Composite Materials - Analysis of Natural and Man-Made Materials. s.I.:InTech.

Hajianmaleki, M. \& Qatu, M. S., 2012. Static and vibration analyses of thick, generally laminated deep curved beams with different boundary conditions.. Composites: Part B, p. 1767-1775.

Kim, J.-G., 2005. An Effective Composite Laminated Curved Beam Element. Communications in Numerical Methods in Engineering, pp. 453-466. 
Kreja, I., 2011. A literature review on computational models for laminated composite and sandwich panels. Central European Journal of Engineering, pp. 59-80.

Nguyen, T., 2010. Effects of Curvature on the Stresses of a Curved Laminated Beams subjected to Bending. Arlington, TX: s.n.

Reddy, J., 1985. Review of the Literature on Finite-Element Modeling of Laminated Composite Plates. s.I.:Journal of Shock and Vibration Digest.

Reddy, J., 1997. Mechanics of Laminated Composite Plates: Theory and Analysis. Boca Raton, Fla: CRC Press,

Sreeram, T. \& Sivaneri, N., 1997. FE-Analysis of a Moving Beam using Lagrangian Multiplier Method. International Journal of Solids and Structures.

Tanov, R. \& Tabiei, A., 2000. A simple correction to the first-order shear deformation shell finite element formulations.. Finite Elements in Analysis and Design, p. 189-197.

Timoshenko, S. P. \& Goodier, J. N., 1970. Theory of Elasticity. In: s.I.:McGraw-Hill Book Company, pp. 83-88.

Tornabene, F., Fantuzzi, N., Viola, E. \& Carrera, E., 2013. Static analysis of doubly-curved anisotropic shells and panels using CUF approach, differential geometry and differential quadrature method. Composite Structures, Issue 107, p. 675-697.

Washizu, K., 1964. Some Considerations on a Naturally Curved and Twisted Slender Beam. s.l.:Journal of Mathematics and Physics. 